\title{
Tidal Response of Mars Constrained from Laboratory-based Viscoelastic Dissipation Models and Geophysical Data
}

\author{
A. Bagheri ${ }^{1}$, A. Khan ${ }^{2,1}$, D. Al-Attar ${ }^{3}$, O. Crawford ${ }^{3}$, D. Giardini ${ }^{1}$ \\ ${ }^{1}$ Institute of Geophysics, ETH Zürich, Zürich, Switzerland \\ ${ }^{2}$ Institute of Theoretical Physics, University of Zürich, Zürich, Switzerland \\ ${ }^{3}$ Bullard Laboratories, Department of Earth Sciences, University of Cambridge, Cambridge, UK
}

\section{Key Points:}

- We present a method for determining the planetary tidal response using laboratorybased viscoelastic models and apply it to Mars.

- Maxwellian rheology results in considerably biased (low) viscosities and should be used with caution when studying tidal dissipation.

- Mars' rheology and interior structure will be further constrained from InSight measurements of tidal phase lags at distinct periods.

Corresponding author: Amirhossein Bagheri, amirhossein. bagheri@erdw.ethz.ch 


\begin{abstract}
We employ laboratory-based grain-size- and temperature-sensitive rheological models to describe the viscoelastic behavior of terrestrial bodies with focus on Mars. Shear modulus reduction and attenuation related to viscoelastic relaxation occur as a result of diffusionand dislocation-related creep and grain-boundary processes. We consider five rheological models, including extended Burgers, Andrade, Sundberg-Cooper, a power-law approximation, and Maxwell, and determine Martian tidal response. However, the question of which model provides the most appropriate description of dissipation in planetary bodies, remains an open issue. To examine this, crust and mantle models (density and elasticity) are computed self-consistently through phase equilibrium calculations as a function of pressure, temperature, and bulk composition, whereas core properties are based on an Fe-FeS parameterisation. We assess the compatibility of the viscoelastic models by inverting the available geophysical data for Mars (tidal response and mean density and moment of inertia) for temperature, elastic, and attenuation structure. Our results show that although all viscoelastic models are consistent with data, their predictions for the tidal response at other periods and harmonic degrees are distinct. The results also show that Maxwell is only capable of fitting data for unrealistically low viscosities. Our approach can be used quantitatively to distinguish between the viscoelastic models from seismic and/or tidal observations that will allow for improved constraints on interior structure (e.g., with InSight). Finally, the methodology presented here is generally formulated and applicable to other solar and extra-solar system bodies where the study of tidal dissipation presents an important means for determining interior structure.
\end{abstract}




\section{Plain Language Summary}

A planet responds to external tidal forces, such as those created by an orbiting moon, by deforming, which causes a change in its external gravitational potential field. If the body responds elastically, the tide raised on the planet by its moon will be aligned with the tide-raising potential as a result of which there will be no dissipation of energy within the planet. However, ordinary planetary materials respond anelastically, which means that energy is being dissipated and, consequently, the tidal bulge will be misaligned with the tide-raising moon. The amount by which a planetary body responds to an external tidal force depends on its interior structure such that rigid bodies will not deform appreciably, whereas less rigid bodies can deform significantly. Here, we use this observation for the Mars-Phobos system to constrain the interior structure of Mars. The models that describe the planet's response to an external force are based on laboratory measurements of the deformation of major planetary materials. The Mars InSight mission will make further measurements of the tidal response of Mars for comparison with our modeling results, which will improve our understanding of Mars's interior structure and dynamical evolution. 


\section{Introduction}

A planet responds to tidal forces by deforming, which causes a change in its gravitational potential field (see Figure 1). If the response is purely elastic, the tide raised on the planet by its moon, and vice versa, will be aligned with the tide-raising potential as a result of which the orbit of the moon will be unaffected, i.e., there is no torque acting and no dissipation occurs within either body. If, however, the planet reacts anelastically, dissipation is acting, as a result of which the tidal bulge and the tide-raising potential are misaligned. Since the tidal bulge reacts by applying a torque, which is proportional to the amplitude of the tide and to the sine of the tidal lag angle or phase lag, the orbit of the moon changes. Consequently, the phase lag is a measure of tidal dissipation and is determined from the angle between the tide-raising force and the tide itself and depends on the anelastic structure, whereas the amplitude of the tidal response is mostly sensitive to the elastic structure. Thus by measuring orbital changes of natural or artificial satellites around planets or landed spacecraft, information on a planet's interior structure can be derived as has been done for the terrestrial solar system planets and the Moon [e.g., Padovan et al., 2013; Efroimsky and Lainey, 2007; Hauck et al., 2013; Yoder, 1995; Konopliv and Yoder, 1996; Rivoldini et al., 2011; Bills et al., 2005; Khan and Connolly, 2008; Williams et al., 2006; Nimmo et al., 2012; Nimmo and Faul, 2013; Dumoulin et al., 2017; Williams et al., 2014; Williams and Boggs, 2015; Khan et al., 2018; Zharkov and Gudkova, 2005; Yoder et al., 2003, among others].

The anelastic processes that most solid state materials undergo in response to a forcing are governed by dissipative processes at the microscopic scale, in particular viscoelastic relaxation of the shear modulus due to elastically-accommodated, and dislocation- and diffusion-assisted grain-boundary sliding [Karato and Spetzler, 1990; Ranalli, 2001; Takei et al., 2014; Faul and Jackson, 2015; Karato et al., 2015]. Several models have been proposed to describe the viscoelastic behavior of planetary materials. For example, Maxwell's model, the simplest of all rheological models, has often been called upon when studying tidal dissipation in planets and moons [e.g., Bills et al., 2005; Correia et al., 2014; Remus et al., 2012; Efroimsky and Lainey, 2007]. Yet this model only includes an elastic and a viscous response without a transient regime that, from a time-scale point of view, covers most of the period range of interest where tidal dissipation actually occurs. Also, Maxwell's model has difficulty in reproducing the observed frequency dependence of dissipation $\propto \omega^{-\alpha}$, where $\omega$ is angular frequency and $\alpha$ the frequency exponent [e.g., Minster 
and Anderson, 1981; Jackson et al., 2002; Benjamin et al., 2006; Jackson and Faul, 2010]. As a consequence, Maxwellian rheology results in an unsatisfactory explanation for the tidal response of planetary bodies like Mars, the Moon, and the Earth [Bills et al., 2005; Nimmo et al., 2012; Nimmo and Faul, 2013; Williams and Boggs, 2015; Renaud and Henning, 2018; Lau and Faul, 2019].

In response hereto, more complex grain-size- and temperature-dependent models have been proposed. Among these figure the models of Andrade, Burgers, SundbergCooper, and power-law approximation scheme, which have been studied experimentally [Jackson et al., 2002; Sundberg and Cooper, 2010; Jackson and Faul, 2010; Takei et al., 2014; McCarthy et al., 2011; Sasaki et al., 2019]. Laboratory experiments of torsional forced oscillation data on anhydrous melt-free olivine appear to favour the extended Burgers model over other rheological models because of its ability to describe the transition from (anharmonic) elasticity to grain size-sensitive viscoelastic behaviour [Faul and Jackson, 2015]. Because of the improved flexibility that comes with a larger number of degrees of freedom, application of these laboratory-based dissipation models to geophysical problems has nonetheless resulted in considerable improvement in matching the observed frequency dependence of dissipation, in addition to simultaneously fitting attenuationrelated data that span the frequency range from the dominant seismic wave period $(\sim 1 \mathrm{~s})$ over normal modes ( $\sim 1$ hour) to the very long-period tides ( $\sim 20$ years), i.e., a frequency range spanning 5 orders of magnitude [Henning et al., 2009; Efroimsky, 2012a,b; Nimmo et al., 2012; Nimmo and Faul, 2013; Khan et al., 2018; Lau and Faul, 2019; Benjamin et al., 2006; Renaud and Henning, 2018].

While qualitatively similar in that the various viscoelastic models can be described in terms of dashpot and spring elements that are arranged in series and parallel, it is yet to be understood to what extent these models are quantitatively similar on planetary scales, i.e., are capable of making predictions that match global geophysical observations at different forcing frequencies for a set of realistic models of the interior structure of planets. While most studies focus on application of a single viscoelastic dissipation model to solar system objects: Mercury [Padovan et al., 2013], Venus [Dumoulin et al., 2017], Earth [Bellis and Holtzman, 2014; Abers et al., 2014; Agnew, 2015; Karato et al., 2015; Lau and Faul, 2019], the Moon [Nimmo et al., 2012; Efroimsky, 2012a,b; Karato, 2013; Harada et al., 2014; Williams and Boggs, 2015; Qin et al., 2016], Mars [Lognonné and Mosser, 1993; Yoder et al., 2003; Sohl et al., 2005; Zharkov and Gudkova, 2005; Bills et al., 2005; 
Efroimsky and Lainey, 2007; Nimmo and Faul, 2013; Khan et al., 2018], Io [Hussmann and Spohn, 2004; Bierson and Nimmo, 2016; Renaud and Henning, 2018], Iapetus [Peale, 1977; Robuchon et al., 2010; Castillo-Rogez et al., 2011], Europa [Moore and Schubert, 2000; Hussmann and Spohn, 2004; Wahr et al., 2009; A et al., 2014], Ganymede [A et al., 2014; Kamata et al., 2016], Enceladus [Roberts and Nimmo, 2008; Choblet et al., 2017], and exoplanets [Henning et al., 2009; Efroimsky, 2012b; Renaud and Henning, 2018], studies that quantitatively investigate several viscoelastic models concomitantly by formulating the problem in a geophysical inverse sense have yet to be undertaken.

With this in mind, we consider a series of laboratory-based viscoelastic dissipation models and quantitatively compare them using geophysical inversion with the purpose of constraining attenuation properties of planets from seismic to tidal time scales. Here, we focus on Mars for which the tidal response due to Phobos (amplitude and phase lag), in addition to mean density and mean moment of inertia, are available. The approach adopted here builds upon and extends previous work [e.g., Renaud and Henning, 2018; Khan et al., 2018] in that it seeks to combine a suite of experimentally-constrained grain size-, temperature- and frequency-dependent viscoelastic models (Andrade, extended Burgers, Sundberg-Cooper, Maxwell, and a power-law approximation scheme) with petrologic phase equilibrium computations that enables self-consistent computation of geophysical responses for direct comparison to observations. The advantage of this approach is that it anchors internal structure parameters that are in laboratory-based models, while geophysical inverse methods are simultaneously employed to optimise profiles of e.g., seismic wave speeds, dissipation, and density to match a set of geophysical observations.

Quantitative predictions of e.g., the tidal response at different periods can be made and tested against results that are expected to be obtained from the Mars InSight (Interior Exploration using Seismic Investigations, Geodesy and Heat Transport) mission, which has been operating on Mars for eight months since its deployment. InSight will measure attenuation, with both the SEIS (Seismic Experiment for Internal Structure) [Lognonné, 2019] and RISE (Rotation and Interior Structure Experiment) [Folkner et al., 2018] instruments at periods ranging from seconds (seismic events) to months (nutation and precession of MarsâĂŹs rotation axis). The observation of attenuation at periods other than the main Phobos tide provides a means for distinguishing between the various laboratorybased dissipation models and will turn out to be of particular importance for understanding the thermal and viscoelastic behaviour of Mars. For community use, we tabulated pre- 


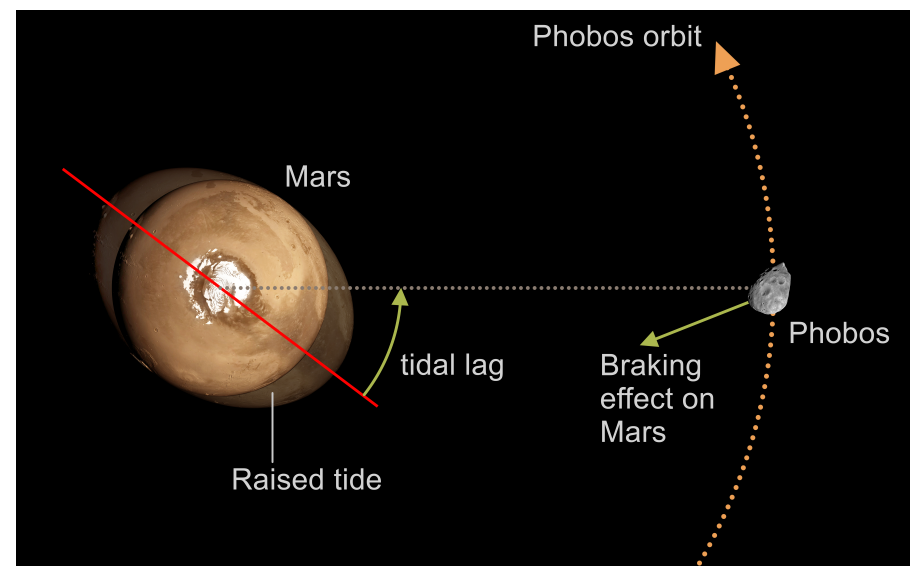

Figure 1. Illustration of the tidal interaction between Mars and its larger moon Phobos. Courtesy of David Ducros/IPGP.

dicted model responses (Love numbers and attenuation) at a number of distinct periods and spherical harmonic degree for each of the rheological models considered here. Finally, we would like to note that although this study focuses on Mars, the methodology described herein is generally applicable and is easily extendable to other solar system bodies and beyond.

\section{Background}

\subsection{Geophysical Analysis}

The tidal bulge raised on Mars (see Figure 1) due to its orbiting moons Phobos and Deimos, is a function of its internal structure and the forcing itself. Because dissipation is acting, the bulge does not align with the barycenteric axis (defined as the line that extends between the center of masses of the two objects and indicated by the dashed line in Figure 1) but is lagging behind Phobos and ahead of Deimos. As a result of the tidal bulge, changes in the potential field and deformations in both radial and tangential directions of Mars ensue (the same holds for the moons). The change in the potential field of a planet of radius $r$, subjected to a perturbation in potential $\Phi$ due to an orbiting moon is denoted by $\phi$, and can be expressed as a spherical harmonics expansion in time domain as (in what follows we rely on the formulation of Efroimsky and Makarov [2014])

$$
\phi_{n}(R, t)=k_{n}\left(\frac{R}{r}\right)^{n+1} \Phi_{n}\left(R, R^{*}\right)
$$


where $n$ indicates the spherical harmonic degree, $k_{n}$ is the potential Love operator of degree $n, R^{*}$ is the position of the perturbing body, and $R$ is a point on Mars's surface. The displacement Love operators, $h_{n}$ and $l_{n}$ express the resultant vertical (radial) and horizontal (tangential) displacements at the surface of the planet as $h_{n} \Phi_{n} / g$ and $l_{n} \nabla \Phi_{n} / g$, respectively, where $g$ is the gravitational acceleration at the surface. In addition to the Love numbers, the magnitude of the change in gravity due to the change in the potential field is of interest. This parameter, the gravimetric factor $\delta$, is computed as $\delta_{n}=$ $1+2 h_{n} / n-k_{n}(n+1 / n)$ [e.g., Agnew, 2015].

In the frequency domain, equation 1 can be written as

$$
\phi_{n}\left(R, \omega_{p q}^{n m}\right)=\left(\frac{R}{r}\right)^{n+1} \bar{k}_{n}\left(\omega_{p q}^{n m}\right) \bar{\Phi}_{n}\left(R, R^{*}, \omega_{p q}^{n m}\right),
$$

where, $\omega_{p q}^{n m}$ are the Fourier tidal modes, $n m$ and $p q$ are integers used to number the modes, and $\bar{k}_{n}$ is the complex frequency-dependent Love number where $\bar{k}_{n}\left(\omega_{p q}^{n m}\right)=\mathfrak{R}\left[\bar{k}_{n}\left(\omega_{p q}^{n m}\right)\right]+$ $i \mathfrak{I}\left[\bar{k}_{n}\left(\omega_{p q}^{n m}\right)\right]$. The Love number $k_{n}$ can be written as $\left|\bar{k}_{n}\right| \exp \left(-i \epsilon_{n}\right)$, where $\epsilon_{n}$ is the phase angle between the tidal force and resulting bulge and equals the geometric lag $\left(\delta_{p q}^{n m}\right)$ (labeled "tidal lag" in Figure 1) through $\delta_{p q}^{n m}=\epsilon_{p q}^{n m} / m$ [e.g., Efroimsky and Makarov, 2013]. The phase angle is also related to the energy that is being dissipated in the tides as $1 / Q_{n}$, where $Q_{n}$ is the tidal quality factor of spherical harmonic degree $n$

$$
Q_{n}=\frac{1}{\sin \left|\left(\epsilon_{n}\right)\right|}=\frac{\sqrt{\mathfrak{R}^{2}\left(k_{n}\right)+\mathfrak{J}^{2}\left(k_{n}\right)}}{\left|\mathfrak{J}\left(k_{n}\right)\right|}
$$

For the terrestrial planets, $\epsilon_{n}$ is usually small at the main tidal periods (except when the satellite is very close to the resonance period), as a result of which $Q_{n}$ can be approximated by

$$
Q_{n} \approx \frac{1}{\tan \left|\left(\epsilon_{n}\right)\right|}=\frac{\mathfrak{R}\left(k_{n}\right)}{\left|\mathfrak{J}\left(k_{n}\right)\right|} .
$$

In the following section, we turn our attention to intrinsic shear attenuation.

\subsection{Viscoelastic Dissipation Models}

While elasticity is a result of bond stretching along crystallographic planes in an ordered solid, viscosity and dissipation inside a polycrystalline material occur by motion of point, linear, and planar defects, facilitated by diffusion. In viscoelastic behavior, each of these mechanisms contribute [e.g., Karato, 2008]. Deformations of a viscoelastic solid depends on the temporal-scale of the applied load [Chawla and Meyers, 1999]. For small stresses, the stress-strain relation is linear, and the response is described in the 
time-domain via the creep function $J(t)$. The creep function links material properties and forcing (input) with the "felt" (relaxed) shear modulus and phase lag due to attenuation (output). The response of the material to forcing consists of an instantaneous elastic response followed by a semi-recoverable transient flow regime where the strain rate changes with time, and finally yields to steady-state creep. Based on this, the general form of the creep function for a viscoelastic solid consists of three terms:

$$
\underbrace{J(t)}_{\text {Creep function }}=\underbrace{J_{U}}_{\text {Elastic }}+\underbrace{f(t)}_{\text {Transient strain-rate }}+\underbrace{t / \eta}_{\text {Viscous }}
$$

where $t$ is time and $\eta$ is the steady-state Newtonian viscosity. The complex shear modulus $\hat{G}$ is computed from the Laplace-transformed creep or the complex compliance $\hat{J}=\mathfrak{R}(\hat{J})+$ $i \mathfrak{J}(\hat{J})$ through $\hat{G}=1 / \hat{J}$ [Findley and Onaran, 1965]. The relaxed shear modulus and the associated dissipation $\left(Q_{\mu}^{-1}\right)$ are obtained from the following expressions:

$$
G_{R}(\omega)=\left\{\mathfrak{R}^{2}[\hat{J}(\omega)]+\mathfrak{J}^{2}[\hat{J}(\omega)]\right\}^{-\frac{1}{2}},
$$

$$
Q_{\mu}^{-1} \approx|\mathfrak{J}[\hat{J}(\omega)]| / \mathfrak{R}[\hat{J}(\omega)] .
$$

Note that $Q_{\mu}$ is an intrinsic material property and therefore different from the global $Q_{n}$ discussed in the previous section (cf. Eq. 3). Briefly, and as discussed in more detail in e.g., Efroimsky [2015] and Lau et al. [2016], the distinction between global tidal dissipation $\left(Q_{n}\right)$ and intrinsic attenuation $\left(Q_{\mu}\right)$, which is a spatially-varying material property and responsible for the attenuation of e.g., seismic waves, derives from the fact that $Q_{n}$, in addition to "sensing" $Q_{\mu}$, is also influenced by gravity and inertial effects due to rotation of the planet. At reasonably high frequencies, this distinction becomes redundant as $Q_{n}$ approaches $Q_{\mu}$.

In the following, we consider a suite of laboratory-based viscoelastic dissipation models: Maxwell, extended Burgers, Andrade, Sundberg-Cooper, and a power-law scheme. These models derive from grain-size, temperature-, and pressure-sensitive viscoelastic relaxation measurements. The dissipation models based on Maxwell, extended Burgers, Andrade, and the power-law scheme are described in detail in Jackson and Faul [2010] and rely on laboratory experiments (temperature range $800-1200^{\circ} \mathrm{C}$ ) of torsional forced oscillation data (period range 1-1000 s) on melt-free poly-crystalline olivine (grain sizes in the range 3-165 $\mu \mathrm{m}$ ). The model of Sundberg and Cooper [Sundberg and Cooper, 2010] is also based on torsional oscillation data, but in a fine-grained (5 $\mu \mathrm{m})$ peridotite 
(olivine +39 vol $\%$ orthopyroxene) specimen (temperature range $1200-1300^{\circ} \mathrm{C}$ and periods of $1-\sim 200 \mathrm{~s})$.

As shown in figure 2, each model can be represented as an arrangement of springs and dashpots connected in series, or in parallel, or a combination of both [Findley and Onaran, 1965; Moczo and Kristek, 2005; Nowick and Berry, 1972; Cooper, 2002; Jackson et al., 2007; McCarthy and Castillo-Rogez, 2013]. The instantaneous elastic response is mimicked by a spring (element $1, \mathrm{E} 1$ ) and the fully viscous behavior by that of a dashpot (element 2, E2). The series connection (i.e., a Maxwell module), includes a nonrecoverable displacement, while a parallel connection (a Voigt module) ensures fully recoverable deformations with either a discrete (element 3, E3) or a continuous distribution (element 4, E4, henceforth "modified" Voigt module) of anelastic relaxation times. These models have been applied in various circumstances to model the response of planetary bodies. In the following, we briefly describe each of these models that are employed later to model tidal dissipation within Mars.

\subsubsection{Maxwell}

Maxwell is the simplest model for expressing the viscoelastic behavior and is a series connection of a spring and dashpot. The associated creep function with this model is:

$$
J(t)=\underbrace{J_{U}}_{\mathrm{E} 1}+\underbrace{\frac{t}{\eta}}_{\mathrm{E} 2} .
$$

Here, $J_{U}$ is the unrelaxed, i.e., infinite-frequency, compliance, and E1 and E2 represent spring and dashpot elements (cf. Figure 2), respectively. The compliance for this model is

$$
\hat{J}=J_{U}-\frac{i}{\omega}
$$

and real and imaginary parts of the complex shear modulus are computed from equation 6

$$
\begin{aligned}
& \mathfrak{R}[\hat{G}(\omega)]=\frac{\tau_{M}^{2} \omega^{2}}{J_{U}\left(\tau_{M}{ }^{2} \omega^{2}+1\right)}, \\
& \mathfrak{I}[\hat{G}(\omega)]=\frac{\tau_{M} \omega}{J_{U}\left(\tau_{M}{ }^{2} \omega^{2}+1\right)},
\end{aligned}
$$

where $\tau_{M}=\eta / G_{U}$ is the Maxwell time, $\omega$ is frequency, and $G_{U}$ is the unrelaxed shear modulus. As is apparent from comparison of equations 6 and 8, this model does not include a transient phase and immediately drops to the viscous fluid regime from the elastic response. Hence, while this model represents a reasonable approximation for very 


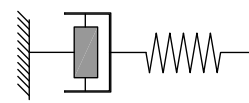

Maxwell

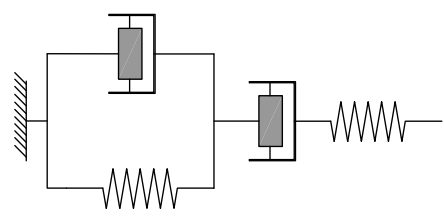

Burgers

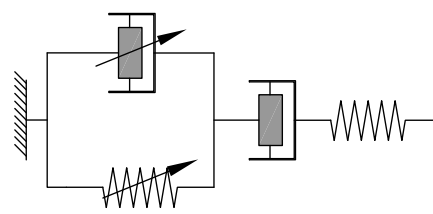

Andrade

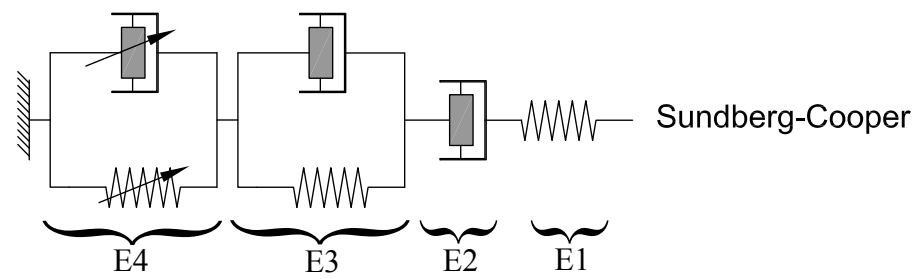

Figure 2. Schematic representation of the viscoelastic models in terms of springs and dashpots. A spring element (E1) represents a purely elastic response, whereas a dashpot element (E2) is representative of purely viscous damping. A series connection of elements 1 and 2 is representative of the response of a Maxwell model (irrecoverable), whereas a connection of elements 1 and 2 in parallel (element 3 ) results in an anelastic (recoverable) response with a discrete (single) spectrum of relaxation times. Arrows on spring and dashpot in element 4, conversely, indicate an element that incorporates a continuous distribution of anelastic relaxation times and results in a broadened response spectrum. Modified from Renaud and Henning [2018]. 
long-period loading such as glacial isostatic adjustments [Peltier, 1974], it does not suffice for modeling the viscoelastic behaviour at intermediate periods. An extended form of Maxwell's model is employed in this study, where effects of grain size, temperature, and pressure are accounted for through a modification of the Maxwell time $\left(\tau_{M}\right)$ [e.g., Morris and Jackson, 2009; Jackson and Faul, 2010; McCarthy et al., 2011] according to

$$
\tau_{M}(T, P, d)=\tau_{M 0}\left(\frac{d_{g}}{d_{0}}\right)^{m_{g v}} \exp \left[\left(\frac{E^{*}}{R}\right)\left(\frac{1}{T}-\frac{1}{T_{0}}\right)\right] \exp \left[\left(\frac{V^{*}}{R}\right)\left(\frac{P}{T}-\frac{P_{0}}{T_{0}}\right)\right],
$$

where $R$ is the gas constant, $E^{*}$ is activation energy, $V^{*}$ is activation volume, $m_{g v}$ is grain size exponent for viscous relaxation, $P$ is pressure, $T$ is temperature, and $\tau_{M 0}$ is a normalized value at a particular set of reference conditions $\left(d_{0}, P_{0}\right.$, and $\left.T_{0}\right)$. Parameter values used here and in the following are tabulated in Table A.1.

\subsubsection{Extended Burgers}

The shortcoming of Maxwell's model in representing a transient response between elastic and viscous regimes can be rectified by introducing a time-dependent anelastic transition between these two regimes. This implies connecting a Voigt module (E3) and a Maxwell module (E1 and E2 connected in series) as shown in Figure 2. For this model, the creep function takes the form

$$
J(t)=\underbrace{J_{U}}_{\mathrm{E} 1}+\underbrace{\Delta J\left[1-\exp \left(-\frac{t}{\tau}\right)\right]}_{\mathrm{E} 3}+\underbrace{\frac{t}{\eta}}_{\mathrm{E} 2},
$$

where E3 corresponds to the anelastic time-dependent response, $J_{U}$ is, as before, unrelaxed compliance, respectively, $\Delta J$ is the magnitude of the anelastic contribution, and $\tau$ is the time constant for the development of the anelastic response. More generally, the single anelastic relaxation time $\tau$ can be replaced by a distribution $D(\tau)$ of relaxation times over an interval specified by upper $\left(\tau_{H}\right)$ and lower bounds $\left(\tau_{L}\right)$ [Jackson and Faul, 2010]. From a micromechanical point of view, this distribution is associated with diffusionally accommodated grain-boundary sliding for which dissipation varies monotonically with temperature and period. The creep function of the material takes the form

$$
J(t)=J_{U}\left[1+\Delta \int_{\tau_{L}}^{\tau_{H}} D(\tau)\left[1-\exp \left(-\frac{t}{\tau}\right)\right] d \tau+\frac{t}{\tau_{M}}\right],
$$

where $\Delta$ is the fractional increase in compliance associated with complete anelastic relaxation and is called the anelastic relaxation strength. A commonly used distribution of anelastic relaxation times associated with the monotonic background dissipation is the ab- 
sorption band model proposed by Minster and Anderson [1981]

$$
D_{B}(\tau)=\frac{\alpha \tau^{\alpha-1}}{\tau_{H}^{\alpha}-\tau_{L}^{\alpha}}, \quad 0<\alpha<1,
$$

for $\tau_{L}<\tau<\tau_{H}$ and zero elsewhere. Jackson and Faul [2010] found that their experimental data were better fit by including a dissipation peak in the distribution of anelastic relaxation times, which is superimposed upon the monotonic background along with the associated dispersion. This background peak is mostly attributed to sliding with elastic accommodation of grain-boundary incompatibilities [see Takei et al., 2014, for a different view]. The distribution for such a peak is given by

$$
D_{P}(\tau)=\frac{1}{\sigma \tau \sqrt{2 \pi}} \exp \left(\frac{-\ln \left(\frac{\tau}{\tau_{P}}\right)}{2 \sigma^{2}}\right) .
$$

With this, the components of the dynamic compliance become

$$
\mathfrak{R}[\hat{J}(\omega)]=J_{U}\left(1+\Delta \int_{\tau_{L}}^{\tau_{H}} \frac{D(\tau)}{1+\omega^{2} \tau^{2}} d \tau\right)
$$

$$
\mathfrak{J}[\hat{J}(\omega)]=J_{U}\left(\omega \Delta \int_{\tau_{L}}^{\tau_{H}} \frac{\tau D(\tau)}{1+\omega^{2} \tau^{2}} d \tau+\frac{1}{\omega \tau_{M}}\right) .
$$

Note that $\tau_{L}$ and $\tau_{H}$ define the cut-offs of the absorption band, where dissipation is frequencydependent $\left(\propto \omega^{\alpha}\right)$. The lower bound of the absorption band ensures a finite shear modulus at high frequencies and restricts attenuation at these periods.

All involved timescales $\left(\tau_{M}, \tau_{L}, \tau_{H}\right.$, and $\left.\tau_{P}\right)$ are considered to be grain size-, pressure, and temperature-dependent through [Jackson and Faul, 2010]

$$
\tau_{i}(T, P, d)=\tau_{i 0}\left(\frac{d_{g}}{d_{0}}\right)^{m_{g}} \exp \left[\left(\frac{E^{*}}{R}\right)\left(\frac{1}{T}-\frac{1}{T_{0}}\right)\right] \exp \left[\left(\frac{V^{*}}{R}\right)\left(\frac{P}{T}-\frac{P_{0}}{T_{0}}\right)\right],
$$

where all parameters are as before (cf. Eq 12) and $i=M, L, H, P$. The grain size exponent $m_{g}$ can be different in the case of anelastic $\left(m_{g a}\right.$ for $\left.i=L, H, P\right)$ and viscous relaxation ( $m_{g v}$ for $i=M$ ), respectively. To more realistically account for variations of the unrelaxed shear modulus with temperature and pressure, Jackson and Faul [2010] suggest the following modification

$$
J_{U}(T, P)=\left[G_{U}\left(T_{0}, P_{0}\right)+\left(T-T_{0}\right) \frac{\partial G_{U}}{\partial T}+\left(P-P_{0}\right) \frac{\partial G_{U}}{\partial P}\right]^{-1} .
$$

Values for the temperature and pressure derivatives are given in Table A.1.

\subsubsection{Andrade}

Whereas the extended Burgers model incorporates a distribution of relaxation times within a restricted time-scale to account for the transient anelasic relaxation, Andrade's 
model proposes a distribution of relaxation times in the entire time domain (represented by arrows on spring and dashpot). The resultant configuration of a Maxwell module and a "modified" Voigt module (E4) is illustrated in Figure 2, which results in a creep function of the form [Andrade, 1962]

$$
J(t)=\underbrace{J_{U}}_{\mathrm{E} 1}+\underbrace{\beta t^{\alpha}}_{\mathrm{E} 4}+\underbrace{\frac{t}{\eta}}_{\mathrm{E} 2},
$$

where $\beta$ qualitatively has the same role as $\Delta$ in the extended Burgers model, and $\alpha$ represents the frequency-dependence of the compliance. In this model, the absorption band extends from 0 to $\infty$. This implies that anelastic relaxation effectively contributes across the entire frequency range from short-period seismic waves to geological time-scales. Consequently, Andrade's model is more economically parameterized than the extended Burgers model. Real and imaginary parts of the dynamic compliance are

$$
\mathfrak{R}[\hat{J}(\omega)]=J_{U}\left[1+\beta^{*} \Gamma(1+\alpha) \omega^{-\alpha} \cos \left(\frac{\alpha \pi}{2}\right)\right],
$$

$$
\mathfrak{I}[\hat{J}(\omega)]=J_{U}\left[\beta^{*} \Gamma(1+\alpha) \omega^{-\alpha} \sin \left(\frac{\alpha \pi}{2}\right)+\frac{1}{\omega \tau_{M}}\right],
$$

where $\beta^{*}=\beta / J_{U}$ and $\Gamma$ is the Gamma function. Note that Andrade's model incorporates a broader absorption band (theoretically of infinite width) compared to the extended Burgers model, which ultimately results in frequency-dependent dissipation at all time-scales. Following Jackson and Faul [2010], corrections due to grain size, temperature, and pressure are applied through a pseudo-period master variable, $X$, which replaces the actual period

$$
X=\omega^{-1}\left(\frac{d_{g}}{d_{0}}\right)^{-m_{g}} \exp \left[\left(\frac{-E^{*}}{R}\right)\left(\frac{1}{T}-\frac{1}{T_{0}}\right)\right] \exp \left[\left(\frac{-V^{*}}{R}\right)\left(\frac{P}{T}-\frac{P_{0}}{T_{0}}\right)\right] .
$$

\subsubsection{Sundberg-Cooper}

To model dissipation for the combined effects of diffusional background and elasticallyaccommodated grain-boundary sliding, Sundberg and Cooper [2010] introduce a composite creep function. Their model represents a modification to Andrade's model in order to improve its functionality over a broader frequency range and to account for the variation of the "felt" elastic response as it has to match the unrelaxed compliance $\left(J_{U}\right)$ at high frequencies and the relaxed compliance $\left(J_{R}\right)$ at low frequencies. This model graphically consists of two Voigt modules and a Maxwell module (cf. Figure 2); One module is similar to that used in Andrade's model (E4), whereas the other module is equivalent to that of 
the extended Burgers model (E3). The creep function for the Sundberg-Cooper model is thus

$$
J(t)=\underbrace{J_{U}}_{\mathrm{E} 1}+\underbrace{\delta J\left[1-\exp \left(-\frac{t}{\tau}\right)\right]}_{\mathrm{E} 3}+\underbrace{\beta t^{\alpha}}_{\mathrm{E} 4}+\underbrace{\frac{t}{\eta}}_{\mathrm{E} 2},
$$

where all variables are as before. Similar to what has been implemented in the extended Burgers model, the corresponding term (E3 in Eq. 25), can be replaced by an integral specifying a distribution of anelastic relaxation times $\tau$ as prescribed by Eq. 14 and modifications for grain size, temperature, and pressure are allowed for through equation 19. Also, accounting for the influence of these parameters in the "modified" Voigt module (E4 in Eq. 25) is implemented in a similar fashion to Andrade's model through the pseudoperiod master variable $X$ (Eq. 24). With this in mind, the real and imaginary parts of the dynamic compliance for Sundberg-Cooper's model are:

$$
\mathfrak{R}[\hat{J}(\omega)]=J_{U}\left[1+\beta^{*} \Gamma(1+\alpha) \omega^{-\alpha} \cos \left(\frac{\alpha \pi}{2}\right)+\Delta \int_{\tau_{L}}^{\tau_{H}} \frac{D(\tau)}{1+\omega^{2} \tau^{2}} d \tau\right],
$$

$$
\mathfrak{J}[\hat{J}(\omega)]=J_{U}\left[\beta^{*} \Gamma(1+\alpha) \omega^{-\alpha} \sin \left(\frac{\alpha \pi}{2}\right)+\omega \Delta \int_{\tau_{L}}^{\tau_{H}} \frac{\tau D(\tau)}{1+\omega^{2} \tau^{2}} d \tau+\frac{1}{\omega \tau_{M}}\right] .
$$

\subsubsection{Power-law Approximation}

As a final model, we consider a power-law approximation, which was originally proposed as a means of fitting earlier measurements [Jackson et al., 2002]. This model is not based on physical principles, but merely represents an approximation of shear dissipation. This power-law scheme requires that $Q_{\mu}^{-1} \ll 1$. Similar to the Andrade and SundbergCooper models, this model also employs a pseudo-period master variable to account for the effects of temperature, pressure, and grain size, defined similar to $X$ in Eq. 24 with $m_{g}$ $=1$ [Jackson and Faul, 2010]. The power-law for $Q_{\mu}$ takes the form

$$
Q_{\mu}^{-1}=A X^{\alpha},
$$

where $A$ is the power-law coefficient. The shear modulus dispersion associated with this dissipation model is

$$
\frac{G(\omega)}{G_{U}}=1-\cot \left(\frac{\alpha \pi}{2}\right) Q_{\mu}^{-1}(\omega) .
$$

\subsection{Comparing the sensitivity of the rheological models}

Before applying the aforementioned dissipation models to Mars, it would be informative to consider the sensitivity of intrinsic material properties to a number of key vari- 
ables. Here, we focus on the dispersion of shear modulus $G_{R}$ and attenuation factor $Q_{\mu}$ with forcing period, temperature, and grain size (all at constant pressure), which is shown in Figure 3. All parameter values used to compute the response curves are compiled in Table A.1. First off, we notice that both $G_{R}$ and $Q_{\mu}$ vary considerably within the range of forcing periods considered here, which includes the tidal forcing periods of the Sun and Phobos and those of long- and short-period seismic waves (vertical lines on Figure 3a and Figure 3b). Most of the short-period seismic band (periods $<1 \mathrm{hr}$ ) is governed by a broad, low-relaxation strength, high-frequency plateau (arrow in Figure 3b), characteristic of elastically-accommodated grain-boundary sliding (E3 in Figure 2), which for tidal periods ( $>1 \mathrm{hr}$ ) gives way to a continuous distribution of anelastic relaxation times characteristic of the high-temperature background (E4 in Figure 2). It has to be noted though that the exact location (in time) of the various processes is currently not well resolved. In general, the same features are observed in the plots showing temperature variations (Figure 3, plots $\mathrm{c}$ and d) in most of the ranges of interest for tidal studies. In the range of high $Q_{\mu}$, i.e., short periods, low temperatures, and large grain sizes, the behaviour of the extended Burgers and Sundberg-Cooper models is due to the existence of a background dissipation peak (less apparent) associated with elastically-accommodated grain-boundary sliding (E3), which occurs around 1300-1400 K, although the interpretation of the background peak is less clear and is currently unexplained by any existing model [Takei et al., 2014; Raj and Ashby, 1975; Gribb and Cooper, 1998]. Based on the relative variation of the response curves, we would expect to see little difference between the Andrade, extended Burgers, and Sundberg-Cooper models. Seismically, i.e., in terms of the relaxed shear modulus behaviour, Andrade and the extended Burgers models are similar as expected based on Figure 2, while the response of the Sundberg-Cooper model is expected to be slightly different in the seismic band.

Relative to forcing period and temperature, $Q_{\mu}$ appears to vary little with grain size (Figure 3, plot e), whereas $G_{R}$ undergoes significant changes for very small grain sizes $(<0.1 \mathrm{~mm})$ (Figure 3, plot f). In contrast, the largest changes in $Q_{\mu}$ occur in the range of relatively large grain sizes $(10-100 \mathrm{~mm})$ and, because of the relative flatness of the extended Burgers and Sundberg-Cooper models in this range, compared to Andrade and and power-law, respectively, the latter two are more likely to resolve (large) grain sizes. Also, since small grain sizes are accompanied by a considerable reduction in $G_{R}$, which is equivalent to an overall "softening", and, as a consequence, a potentially significant 
change in tidal response, small grain sizes are less likely to accord with observations. Incidentally, the grain-size insensitivity of the extended Burgers model, in addition to preferential sampling of relative large grain sizes, was observed in our previous work [Khan et al., 2018].

It is readily recognized from this comparison that the behaviour of Maxwell's model is distinct. In fact, the aforementioned lack of a transient response from elastic to viscous behaviour is clearly visible in Figure 3 as a sudden drop-off in $G_{R}$. While the Maxwell model clearly shows evidence of frequency-dependent dissipation, the latter is too strong to be representative of dissipation in planetary materials. As indicated in Figure 3, the tidal periods of Mars lie in the intermediate range, where a composite of both elastic and viscous regimes contribute to the response - a feature that is incompatible with Maxwell's model. This will be discussed further in section 5.2.4. As for the power-law, the other simplified rheological model, it shows behaviour that appears compatible with the three main models in the restricted range of low temperatures, seismic periods $(\sim 1 \mathrm{~s}-30 \mathrm{~min})$, and larger grain sizes. However, since this model, like Andrade, lacks a cut-off in the frequency-dependent absorption band, both show similar behaviour in the aforementioned parameter range.

As a preliminary summary, we can make the following predictions: 1) the response of Maxwell's model is such that it is unlikely to match geophysical observations throughout most of the period range of interest; 2) the long-period and high-temperature behaviour of the power-law scheme is not realistic; 3) the Andrade, extended Burgers, and SundbergCooper models provide qualitatively similar responses over most of the period and temperature range considered here, although Andrade, as expected, is less dissipative at the very longest periods and highest temperatures. The similarity of the three models is not unsurprising given that they contain many of the same elements as shown in Figure 2. These observation will be quantitatively assessed in the following, where the laboratory-based dissipation models are combined with geophysical inverse modeling.

\section{Geophysical data}

In this study we focus on mean density $(\bar{\rho})$, normalized mean moment of inertia $\left(I / M R^{2}\right)$, and tidal response in the form of the second-degree tidal Love number $\left(k_{2}\right)$ and global tidal dissipation or tidal quality factor $\left(Q_{2}\right)$. The data are discussed in detail in the literature [e.g., Yoder et al., 2003; Lainey et al., 2007; Konopliv et al., 2016; Genova et al., 


\begin{tabular}{llll} 
Quantity & Symbol & Value and Uncertainty & Reference \\
\hline Mean density & $\bar{\rho}$ & $3935 \pm 1.2 \mathrm{~kg} / \mathrm{m}^{3}$ & Rivoldini et al. [2011] \\
Mean moment of inertia & $I / M R^{2}$ & $0.36379 \pm 0.0001$ & Konopliv et al. [2016] \\
Tidal Love number & $k_{2}$ & $0.169 \pm 0.006$ & Konopliv et al. [2016] \\
Global quality factor & $Q_{2}$ & $95 \pm 10$ & Khan et al. [2018] \\
Mass & $M$ & $6.417 \cdot 10^{23} \pm 2.981 \cdot 10^{19} \mathrm{~kg}$ & Konopliv et al. [2016] \\
Radius & $R$ & $3389.5 \mathrm{~km}$ & Seidelmann et al. [2002]
\end{tabular}

Table 1. Martian geophysical data, uncertainties, and sources. Tidal Love number and global quality factor are referenced to the main tidal period of Phobos $(5.55 \mathrm{hr})$.

2016; Rivoldini et al., 2011; Nimmo and Faul, 2013; Khan et al., 2018] and need not be repeated here. The geophysical data are summarized in Table 1.

\section{Computational aspects}

Formally, predicting data (d) from a set model parameters (m) is usually written as $\mathbf{d}=\mathrm{g}(\mathbf{m})$, where $\mathrm{g}$ embodies the physical laws that connect $\mathbf{m}$ and $\mathbf{d}$. In the present case, g comprises a set of algorithms $\left(g_{1}, \ldots, g_{4}\right)$ as a result of which $\mathbf{d}=\mathrm{g}(\mathbf{m})$ can be written as

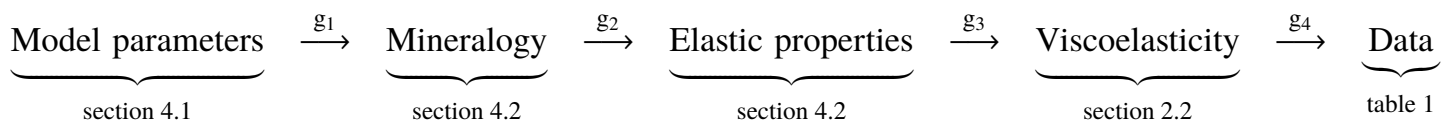

In the following, we describe the steps needed to compute "synthetic" data $\left(\bar{\rho}, I / M R^{2}, k_{2}\right.$, and $Q_{2}$ ) from the model parameters.

\subsection{Model parameterisation and prior model distribution}

We assume a spherically symmetric model of Mars consisting of crust, lithosphere, mantle, and core as illustrated in Figure 4.

Crust and mantle. In line with our previous work [Khan and Connolly, 2008; Khan et al., 2018], crust and mantle compositions are parameterized in terms of major element composition in the model chemical system CFMASNa (comprising the oxides of the elements $\mathrm{CaO}-\mathrm{FeO}-\mathrm{MgO}-\mathrm{Al}_{2} \mathrm{O}_{3}-\mathrm{SiO}_{2}-\mathrm{Na}_{2} \mathrm{O}$ ); a system that accounts for more than $98 \%$ of the mass of Mars' silicate envelope. Crust and mantle compositions are fixed in this study 
and are compiled in Table 3. The crust is further parameterized in terms of thickness and surface porosity. Porosity $\gamma$ is assumed to vary linearly from the surface to the bottom of the Moho (of thickness $d_{\text {crust }}$ ), where porosity vanishes due to pressure. The lithosphere is described by thickness $\left(d_{l i t}\right)$ and temperature $\left(T_{l i t}\right)$. Within the crust and lithosphere, temperature is computed by a linear areothermal gradient that is determined from a fixed surface temperature $\left(T_{\text {surface }}\right)$ and lithospheric temperature and depth. The sublithospheric mantle adiabat is defined by the entropy of the lithology at the temperature $T_{l i t}$ and at depth $d_{l i t}$, which also defines the location where the conductive lithospheric geotherm intersects the mantle adiabat. Mantle viscoelasticity. Parameters needed to compute mantle viscoelasticity depend on the chosen rheological model (section 2.2). The two important parameters that are common to all of the rheological models are grain size $\left(d_{g}\right)$ and frequency-dependence $(\alpha)$. In addition to these two parameters, we consider anelastic relaxation strengths $\Delta_{B}$ and $\beta$ and Andrade-model coefficient $A$ as variable parameters given their importance in determining viscoelastic behaviour. Activation energy $\left(E^{*}\right)$ and volume $\left(V^{*}\right)$ were shown to be of less relevance in our previous work [Khan et al., 2018]. All other viscoelastically-related parameters are fixed and given in Table A.1.

Core. As in most geophysical models of Mars, we assume that $\mathrm{S}$ is the dominant light element 1) because $\mathrm{Si}, \mathrm{C}$, and $\mathrm{O}$ are not sufficiently soluble in an Fe-rich liquid at the low pressures that are expected to have been maintained during core formation [Stevenson, 2001] and 2) because of the observed depletion of chalcophile elements, notably S, of the Martian meteorities [McSween and McLennan, 2014]. Following previous work [e.g., Rivoldini et al., 2011; Khan et al., 2018], the core is assumed to be liquid, convecting, and well-mixed, and parameterised in terms of radius $\left(r_{\text {core }}\right)$, Sulphur content $\left(X_{S}\right)$, and temperature (adiabat). The core adiabat is not independent of the mantle adiabat, but determined so that the thermodynamically-computed temperature at the coremantle-boundary provides the input temperature for the core adiabat.

Finally, all parameters and prior model parameter distributions are summarised in tables $2-4$.

\subsection{Computing elastic and viscoelastic properties}

To compute stable mantle mineralogy, seismic wave velocities, and density along self-consistent mantle adiabats as functions of pressure and composition in the CFMASNa model chemical system, we follow previous work [e.g., Khan and Connolly, 2008; Khan 


\begin{tabular}{llllll} 
Viscoelastic model & \multicolumn{5}{c}{ Parameters and prior information } \\
\hline & $\alpha$ & $d_{g}(\mathrm{~mm})$ & $\beta$ & $\Delta_{B}$ & $A$ \\
\hline Distribution & Uniform & Log-uniform & Log-uniform & Uniform & Uniform \\
\hline Andrade & $0.2-0.6$ & $0.001-50$ & $10^{-14}-10^{-9}$ & - & - \\
Extended Burgers & $0.2-0.6$ & $0.001-50$ & - & $0.9-2$ & - \\
Power-law & $0.2-0.6$ & $0.001-50$ & - & - & $0.001-0.01$ \\
Sundberg-Cooper & $0.2-0.6$ & $0.001-50$ & $10^{-14}-10^{-9}$ & $0.9-2$ & - \\
\hline
\end{tabular}

Table 2. Viscoelastic model parameters and prior distributions.

\begin{tabular}{lll} 
Component & Crust & Mantle \\
\hline $\mathrm{CaO}$ & 7.0 & 2.4 \\
$\mathrm{FeO}$ & 18.8 & 18.7 \\
$\mathrm{MgO}$ & 9.2 & 30.7 \\
$\mathrm{Al}_{2} \mathrm{O}_{3}$ & 10.9 & 3.5 \\
$\mathrm{SiO}_{2}$ & 50.7 & 44.1 \\
$\mathrm{Na}_{2} \mathrm{O}$ & 3.3 & 0.6
\end{tabular}

Table 3. Major element crust and mantle compositions used in this study. Crust and mantle compositions are from Taylor and McLennan [2008] and Taylor [2013]. All numbers in weight percent.

\begin{tabular}{llll} 
Parameter & Description & Interval & Distribution \\
\hline$\gamma$ & Surface porosity & $0.5-0.65$ & Uniform \\
$d_{\text {crust }}$ & Crustal thickness & $10-90 \mathrm{~km}$ & Uniform \\
$Q_{\text {lit }}$ & Shear attenuation in crust and lithosphere & 1000 & fixed \\
$T_{\text {surface }}$ & Surface temperature & $0{ }^{\circ} \mathrm{C}$ & fixed \\
$d_{\text {lit }}$ & Lithospheric depth & $100-400 \mathrm{~km}$ & Uniform \\
$T_{\text {lit }}$ & Lithospheric temperature & $700-1450{ }^{\circ} \mathrm{C}$ & Uniform \\
$r_{\text {core }}$ & Core radius & $0-3000 \mathrm{~km}$ & Uniform \\
$X_{S}$ & Core sulfur content & $0-100 \%$ & Uniform \\
\hline
\end{tabular}

Table 4. Crust, lithosphere, mantle, and core model parameters and prior distributions. 
et al., 2018] and employ Gibbs free energy minimization [Connolly, 2009]. For this purpose, the thermodynamic formulation of Stixrude and Lithgow-Bertelloni [2005b] and parameters of Stixrude and Lithgow-Bertelloni [2011] are used. Pressure is obtained by integrating the surface load. In the context of computing mantle properties, we would like to note that the pressure and temperature derivatives of the shear modulus (Eq. 20) employed earlier (section 2.3), are not used here as these are determined as part of the free energy minimization. To account for the effect of porosity on crustal seismic P- and Swave velocities $\left(\mathrm{V}_{\mathrm{P}}\right.$ and $\left.\mathrm{V}_{\mathrm{S}}\right)$ and density $(\rho)$, all three parameters are multiplied by the depth-dependent porosity.

To compute elastic properties of the core in the FeS system, we rely on the parameterisation of Rivoldini et al. [2011] as also implemented in our previous work [Khan et al., 2018]. Arguments for $\mathrm{S}$ as the main light alloying element in Mars's core are summarised in [e.g., Rivoldini et al., 2011; Khan et al., 2018; Smrekar et al., 2019]. The core is assumed to be homogeneous, fully convecting, and completely molten [e.g., Lognonné and Mosser, 1993; Zharkov and Gudkova, 1997; Yoder et al., 2003]. Since the core is assumed to be fluid, it does not support shear and consequently no shear dissipation occurs. Hence, its response only includes the buoyant component and it is completely in quadrature with the acting force. In line with previous work, bulk dissipation is considered negligible. Finally, to "convert" the elastic (unrelaxed) shear moduli to viscoelastic (relaxed) moduli, we compute shear attenuation $\left(Q_{\mu}\right)$ and relaxed shear moduli using the equations described in section 2.2 for each of the rheological models. Shear attenuation in the crust and lithosphere is fixed to $Q_{l i t}=1000$. As for the core, we assume that dissipation only occurs in shear. This seems appropriate given that dissipation in bulk is negligible [Benjamin et al., 2006].

\subsection{Computing tidal response}

To determine the frequency-dependent tidal response of a spherically symmetric, self-gravitating, and viscoelastic planetary model, we use an adaptation of the method and code developed by Al-Attar and Tromp [2014] and Crawford et al. [2018] for modeling glacial loading. This approach is based on the generalised spherical harmonic expansions (Phinney \& Burridge 1973) of the displacement field and gravitational potential perturbation, and leads to a complete decoupling between the radial expansions coefficients for each spherical harmonic degree and order. The resulting ordinary differential equations 
are then efficiently solved using a one-dimensional spectral element discretisation. Inertial terms in the equations of motion are neglected within these calculations due to the tidal periods being well below those of the gravest free oscillations. Quasi-static deformation in the fluid core is modelled following the approach of Dahlen [1974], with the inclusion of tidal forces requiring a slight modification of the theory as described in appendix B. The resulting code can calculate the Love numbers $k_{n}, h_{n}$, and $l_{n}$ along with the quality factors $Q_{n}$ for any spherical harmonic degree. Mean density and mean moment of inertia are readily obtained from integration of the density profile.

\subsection{Inverse problem}

Following our previous work, the inverse problem $\mathbf{d}=\mathrm{g}(\mathbf{m})$ is solved using a Bayesian approach [e.g., Mosegaard and Tarantola, 1995]

$$
\sigma(\mathbf{m})=\kappa f(\mathbf{m}) \mathcal{L}(\mathbf{m}),
$$

where $\kappa$ is a normalization constant, $f(\mathbf{m})$ is the prior model parameter distribution, $\mathcal{L}(\mathbf{m})$ is the likelihood function, and $\sigma(\mathbf{m})$ is the posterior model parameter distribution and represents the solution to the inverse problem. The form of $\mathcal{L}(\mathbf{m})$ is determined from data, their uncertainties, and data noise modelling (to be described below). To sample the posterior distribution, we employ the Metropolis algorithm, which is an importance sampling algorithm. This stochastic algorithm, which is based on a Markov chain Monte Carlo method, ensures that models that fit data (through $\mathcal{L}(\mathbf{m})$ ) and are consistent with the chosen prior model parameter distribution (through $f(\mathbf{m})$ ) are sampled preferentially.

As concerns the likelihood function, we assume that data noise is Gaussian distributed and that observational uncertainties and modeling errors among the different data sets are independent. As a consequence, the likelihood function takes the form

$$
\mathcal{L}(\mathbf{m}) \propto \prod_{\mathrm{i}} \exp \left(-\frac{\left|\mathrm{d}_{\mathrm{obs}}^{\mathrm{i}}-\mathrm{d}_{\mathrm{cal}}^{\mathrm{i}}(\mathbf{m})\right|^{2}}{2 \sigma_{\mathrm{i}}^{2}}\right),
$$

where the integer $i$ is either $\bar{\rho}, I / M R^{2}, k_{2}$, or $Q_{2}$, and $\mathrm{d}_{\mathrm{obs}}$ and $\mathrm{d}_{\mathrm{cal}}(\mathbf{m})$ refer to observed and calculated "synthetic" data, respectively, and $\sigma$ is the uncertainty associated with each data set. For each rheological model, we sampled around 100,000 models in total and to ensure near-independence, every 20th model was retained for analysis. This number is obtained from analysising the autocorrelation of the liklihood function, which provides a measure of when independence between models has been achieved. 


\section{Results and discussion}

\subsection{Data Fit}

Here and in the following, the main focus will be on the power-law approximation scheme, and the extended Burgers, Andrade, and Sundberg-Cooper rheological models; Maxwell's model will be discussed separately in section 5.2.4. We make this distinction here based on the observation that although Maxwell's model is capable of fitting the observations (not shown), this is only achievable for unrealistically low mean viscosities $\left(\sim 10^{16} \mathrm{~Pa} \cdot \mathrm{s}\right.$, see section5.2.4). The resultant data fits are shown in Figure 5 and indicate that all four rheological models are capable of fitting the observations within uncertainties.

\subsection{Viscoelastic properties}

\subsubsection{Grain size}

The sampled grain-size distributions for each of the rheological models is shown in Figure 6 and indicate that the Andrade, Sundberg-Cooper, and power-law models imply larger grain sizes in comparison to the predictions based on the extended Burgers model. The three former models suggest most probable grain sizes in the range $0.5-4 \mathrm{~cm}$ range, whereas in the case of the latter model, grain sizes are less well-resolved with a slight preference in the range $0.1-1 \mathrm{~cm}$. Importantly, the form of the sampled grain size distributions follows the behaviour observed in Figure 3 closely: Andrade, Sundberg-Cooper, and power-law models show the largest variation in the range $\sim 1-10 \mathrm{~cm}$, while the extended Burgers model is relatively "flat" in the $0.1-10 \mathrm{~cm}$ range, in agreement with our earlier work [Khan et al., 2018].

In general, grain sizes obtained in this study are larger than observed in terrestrial samples, where grains of submillimeter-to-millimeter size are typically found [Karato, 1984]. Incidentally, relatively large grain sizes $(\sim 1-10 \mathrm{~cm})$ are also found in a study by Lau and Faul [2019], where the extended Burgers model was applied to Earth's deep mantle to model its anelastic response (see also section 5.2.2).

In support of larger grain sizes, we showed in our previous work [Khan et al., 2018] how the geophysical results could be employed in tandem with geodynamic simulations to identify plausible geodynamic scenarios and parameters. The geodynamical models were generally able to reproduce the geophysically-determined areotherms, crustal thickness values, and grain sizes, but, in part only, lithospheric thicknesses. Grain sizes greater than 


\begin{tabular}{lllll}
\hline Parameter & Andrade & Extended Burgers & Power-law & Sundberg-Cooper \\
\hline$d_{g}$ & $0.1-2 \mathrm{~cm}$ & $0.01-4 \mathrm{~cm}$ & $0.1-2 \mathrm{~cm}$ & $1-4 \mathrm{~cm}$ \\
$\alpha$ & $0.22-0.38$ & $0.22-0.42$ & $0.22-0.38$ & $0.24-0.38$ \\
$-\log _{10}(\beta)$ & $12.4-13$ & - & - & $13.5-14$ \\
$\Delta_{B}$ & - & $1-1.5$ & - & $1.1-1.4$ \\
$A$ & - & - & $0.0015-0.0025$ & - \\
\hline
\end{tabular}

Table 5. Summary of inversion results for the viscoelastic model parameters considered in this study. Quoted ranges cover the $90 \%$ credible interval.

$1 \mathrm{~mm}$ were mainly restricted to cases of relatively strong grain growth, which tended to increase internal temperature and thicken the lithosphere beyond the current geophysical observations.

For brevity, inversion results for the other viscoelastic model parameters considered here, including frequency exponent $(\alpha)$, anelastic relaxation strengths $\left(\Delta_{B}\right.$ and $\left.\beta\right)$, and power-law coefficient $(A)$, are summarised in Table 5 .

\subsubsection{Temperature and attenuation}

Inverted areothermal and shear attenuation $\left(Q_{\mu}\right)$ profiles are shown in Figure 7 for the major rheological models considered in this study. From this figure, we can make a number of observations. Firstly, the obtained thermal profiles are well-constrained and overlap across the entire depth range. This confirms earlier investigations [Nimmo and Faul, 2013; Khan et al., 2018], where it was shown that global tidal dissipation provides strong constraints on thermal structure. Moreover, the temperature profiles are in good agreement with the results for the extended Burgers model of Khan et al. [2018]. Also, this suggests that the obtained temperature profiles are to first order independent of rheology. Secondly, the shear attenuation profiles overlap in the upper mantle (depth range 200-1000 km), which appears to be highly attenuating with $Q_{\mu}<100$, but differ in the lower part of the mantle (depth range 1000-1600 km), where $Q_{\mu}$ appears to be less constrained for the Andrade and extended Burgers models. Note that although the shear attenuation profiles shown in Figure 7 are computed at the main tidal period of Phobos (5.55 hr), shear attenuation at seismic periods (1 s) are not significantly different with $Q_{\mu}$ 
remaining below 100 for most of the upper part of the mantle (not shown). This suggests that it will be difficult to distinguish between the various rheological models based on the structure of the attenuation profiles. From the point of view of seismology, The implications of this for the propagation and observation of e.g., seismic body and surface waves is such that their detection could be significantly impaired over regional and teleseismic distances. The detection of seismic events by the InSight seismometer [Lognonné, 2019] would therefore present a first-order test of the experimentally-constrained viscoelastic models considered here in the sense that seismic waves that have spent a significant part of their traverse in the mantle from source to station are expected to be attenuated.

\subsubsection{Predicted short- and long-period planetary response}

What the previous discussion suggests is that from knowledge of dissipation at a single frequency (here the main tidal period of Phobos), it appears to be difficult to distinguish between rheological models. If, however, we know the tidal response at other frequencies, more precise arguments can be made about both interior dissipative properties and corresponding rheological models as illustrated in Figure 8 [see also Lognonné et al., 1996; Van Hoolst et al., 2003; Zharkov and Gudkova, 2005; Smrekar et al., 2019]. Figure 8 shows the predicted probability distributions for $k_{2}$ and $Q_{2}$ at three different periods: short- (1 s) and long-period (1 hr) seismic waves, and at the main Solar tide on Mars (12.32 hr) computed for all the inverted models. First off, relative differences in computed $k_{2}$ distributions for the three different periods for a particular rheological model are minor and cover a similar range $\sim 0.16-0.18$ across all theologiealthe models. In the case of $Q_{2}$, however, the distinction within and between models is significantly more pronounced. Although all four rheological models match the only existing observation of $Q_{2}$ at 5.55 hours (Figure 5), they differ in their prediction for $Q_{2}$ at the other periods. In particular, similar behaviour for the Andrade and power-law models, on the one hand, and the extended Burgers and Sundberg-Cooper models, on the other hand, is observed. This "pairing" clearly reflects the common underlying mechanisms that exists between the models. For example, higher dissipation (lower $Q_{2}$ ) at higher frequencies observed for the former two models (Figure $8 \mathrm{c}-\mathrm{d}$ and $\mathrm{g}-\mathrm{h}$ ) is attributed to the presence of the extra dissipation peak, which tends to flatten the $Q_{\mu}$ curves and, as a result, prevents a dramatic increase of attenuation at short time-scales. In contrast, since the frequency-dependent absorption band extends throughout the entire spectrum in the case of Andrade and the power-law 
scheme, low attenuation (high $Q_{2}$ ) at high frequencies ensues (Figure 8a-b and e-f). Note that, although intrinsic attenuation $\left(Q_{\mu}\right)$ plays a key role in determining the tidal quality factor $\left(Q_{2}\right)$, they are not the same. As emphasised, the discrepancy is due to the role of the restoring force of gravity, which increases in importance with increasing forcing period, but is less relevant in the case of seismic waves. Clearly, observations of dissipation at other periods, hold the potential of strongly constraining the anelastic structure.

This is further quantified in Figure 9, which shows the degree-two global response of Mars in the form of $k_{2}, Q_{2}$, and $\delta_{2}$ over a much larger period range ( 1 s-10 yr) for a single inverted model (maximum likelihood model for each rheology). The $Q_{2}$ response behaviour (Figure 9b) for the Andrade and power-law models appears to be dominated by the absorption band with a negative period-dependence, which, in the case of Andrade, slowly transitions into viscous dissipation for periods $>1$ month up until a peak value is reached (not shown) after which friction occurs purely viscously [see also discussion in Efroimsky, 2012a]. As expected, the power-law scheme fails to propose realistic values of $Q_{2}$ at long periods (Figure 9b), which indicates that the Chandler wobble analysis by Zharkov and Gudkova [2009] (with a period of 200 days) that relies on this particular rheological model needs to be reassessed.

In comparison, the response of the extended Burgers and Sundberg-Cooper models is more complex with a broad plateau extending from the seismic into the tidal range that merges into the absorption band with negative frequency dependence (note that the slopes determined by $\alpha$, i.e., the frequency exponent, between the red and black lines are different because the inverted values for $\alpha$ differ for the two models). On the smaller-period side of the plateau, dissipation varies with a positive frequency-dependence, whereas toward the long-period end of the response curves ( $>2 \mathrm{yr}$ ), purely viscous dissipation predominates. For the particular models shown here, Phobos' tide falls in the absorption band in the case of the extended Burgers model, but appears within transition the plateau and the absorption band in the Sundberg-Cooper model. It has to be emphasised though that the relative location of the various features that dominate dissipation at different timescales (see section 2.1) are not well-constrained from the observation at a single period. In summary, this figure serves to indicate that the predicted response behaviour is such that from comparison of a single measurement by InSight of $Q_{2}$ above or below and/or $k_{2}$ below the main tidal period of Phobos, strong constraints on interior structure and dissipative properties can be obtained. 
This has been discussed in terrestrial and lunar studies, where data at different periods are available [e.g., Benjamin et al., 2006; Nimmo et al., 2012; Efroimsky, 2012a; Karato, 2013; Williams and Boggs, 2015; Lau and Faul, 2019]. For example, Lau and Faul [2019] considered seismic normal mode and short- and long-period tidal dissipation measurements for the Earth in an attempt to reconcile the anelastic response of the deep mantle across timescales from $\sim 500 \mathrm{~s}$ to $18.6 \mathrm{yr}$. As briefly indicated earlier, the authors use the extended Burgers model and vary a number of parameters related hereto (e.g., grain size, anelastic relaxation strengths, activation energy and volume, and mantle potential temperature). The authors find that two different frequency dependencies are needed to fit normal mode and tide data. Qualitatively, the authors observe the same anelastic behaviour discussed in relation to the extended Burgers model investigated here (red line in Figure 9), including the presence of a plateau that determines dissipation for periods below $\sim 12 \mathrm{hr}$ and an absorption band above, extending to $\sim 20 \mathrm{yr}$ without clear indication of onset of viscous dissipation. As is the case for our models, the exact occurence of the various characteristics (e.g., plateau, transition to absorption band, and $\alpha$ ) is less wellconstrained.

Finally, we have made model predictions by computing responses at four periods $\left(1 \mathrm{~s}, 1 \mathrm{hr}, 5.55 \mathrm{hr}\right.$, and $12.32 \mathrm{hr}$ ) for all Love numbers $\left(k_{n}, h_{n}\right.$, and $\left.l_{n}\right)$, gravimetric factors $\delta_{n}$, and quality factors $Q_{n}$, for the maximum likelihood models of each rheology and for $n=2-5$. The results are compiled in Table 6. The absolute value of $Q_{n}$ decreases, i.e., dissipation increases, as $n$ becomes larger. This reflects an increased sensitivity to shallower structure, which implies that more of the dissipative part of the planet (mantle) is "seen" with increased spherical harmonic degree. The values obtained here are in good agreement with model predictions made elsewhere [e.g., Van Hoolst et al., 2003; Zharkov and Gudkova, 1997, 2005, 2009]. Based on the observed variation in predicted model values (Figure 9), the phase lags $Q_{n}$ are likely to be much better at discriminating between different models than are the gravimetric factors $\delta_{n}$. This important finding can be examined by the measurements of dissipation provided by both RISE and SEIS. Although beyond the scope of this study, knowledge of higher-degree harmonics are important for modeling e.g., the orbital evolution and future demise of Phobos [Burns, 1978; Efroimsky and Lainey, 2007; Black and Mittal, 2015; Rosenblatt et al., 2016]. 


\subsubsection{Maxwell's Model}

While Maxwell's model, in spite of its simplicity, is capable of fitting data within uncertainties (not shown in Figure 5) for interior structure models that match the results of the other models (see Table 7), this is only possible for very low average viscosities $\left(\sim 2 \cdot 10^{16} \mathrm{~Pa} \cdot \mathrm{s}\right)$ that are well below what is expected for the viscosity of the upper mantle of the Earth $\left(10^{19}-10^{22}\right.$ Pa.s) [e.g., Peltier, 1974; Forte and Mitrovica, 2001; Soldati et al., 2009; Cathles, 2015] and therefore probably unrealistic.

Low mantle viscosities have also been obtained in previous studies [e.g., Bills et al., 2005], where Maxwell's model was applied to estimate the tidal response of Mars. For a homogeneous solid model of Mars, Bills et al. [2005] found an average viscosity of $\sim 10^{15} \mathrm{~Pa} \cdot \mathrm{s}$. Bills et al. [2005] argued that the presence of a liquid core could provide a possible explanation for the low viscosity, but the modeling results based on Maxwell presented here invalidate this inasmuch as a model including a fully liquid core still results in a low average viscosity. We attribute the unrealistically low viscosity values obtained from Maxwell's model to its shortcoming, particularly lack of an intermediate-stage anelastic transient response as also observed elsewhere [e.g., Castillo-Rogez and Banerdt, 2012]. In this context, Castillo-Rogez and Banerdt [2012] found that anelastic transient relaxation processes are required to properly account for Mars's high tidal dissipation. Considering an Andrade rheology and a Mars model with fluid-outer and solid-inner core radii of $1700 \mathrm{~km}$ and $1100 \mathrm{~km}$, respectively, they obtained more "realistic" mantle viscosities of $10^{19}-10^{22} \mathrm{~Pa} \cdot \mathrm{s}$ depending on the assumed value for $\alpha$ (higher $\alpha$ results in lower $\eta$ ).

\subsection{Interior structure}

Since this study focuses on modeling and understanding the anelastic response of Mars at tidal and seismic frequencies, we only briefly summarise the results on interior structure. Inverted model parameters are presented in Table 7 and profiles of P- and Swave speed and density are shown in Figure C.1. While the results for the viscoelastic models largely overlap, it is more difficult to use the results as a means of distinguishing between rheological models with the exception of Maxwell's model. Not unsurprisingly, the results are in good agreement with those of our previous work [Khan et al., 2018], where the influence of compositional parameters was considered in detail in the context of an extended Burgers viscoelastic model. Here as there, models imply relatively large cores ( $\sim 1750-1850 \mathrm{~km}$ in radius) with a significant complement of $\mathrm{S}(\sim 17-20 \mathrm{wt} \%)$. As the 
core $\mathrm{S}$ content found here is close to the eutectic composition and core-mantle-boundary temperatures and pressures are in excess of $1800 \mathrm{~K}$ and $~ 19-20 \mathrm{GPa}$, respectively, a solid inner core is unlikely to be present [e.g., Stewart et al., 2007; Helffrich, 2017]. Moreover, a large core implies that the counterpart of a terrestrial bridgmanite-dominated lower mantle in Mars is unlikely to be present with potentially important implications for the dynamic evolution of Mars's mantle [e.g., Breuer et al., 1997; van Thienen et al., 2006; Ruedas et al., 2013]. For further discussion of interior structure, we refer the reader to previous work [e.g., Rivoldini et al., 2011; Nimmo and Faul, 2013; Plesa et al., 2016; Khan et al., 2018; Smrekar et al., 2019].

\section{Discussion and Conclusion}

In this study, we have examined the geophysical implications of a series of grainsize-, temperature- and frequency-dependent laboratory-based viscoelastic models. These models have been developed in an attempt to describe dissipative properties of planetary materials on the macroscopic scale in terms of interactions that occur on the microscopic scale, i.e., on the level of atoms and grains. The rheological models are based on deformation experiments of melt-free polycrystalline olivine and an olivine-pyroxene mixture, respectively, and include Maxwell, Andrade, extended Burgers, Sundberg-Cooper, and a power-law scheme.

We combined the viscoelastic models with phase equilibrium computations to allow for self-consistently constructed models of seismic elastic and anelastic properties and tested the resultant models against global geophysical observations for Mars. All of the models were found to be able to match the Martian observations including tidal response (amplitude and phase) and mean mass and moment of inertia. The simplest of the investigated rheological models, that of Maxwell, whose response only consists of a purely elastic and a viscous component, only matched the observations for very low viscosities $\left(\sim 10^{16} \mathrm{~Pa} \cdot \mathrm{s}\right)$. This observation is in accord with previous work, where similar results were obtained. Based on the observation that the main tidal periods of most solar system objects are to be found in the transient period range where Maxwell is singularly deficient, it appears reasonable to conclude that Maxwell's model should be abandoned in favour of more realistic models such as Andrade, extended Burgers, or Sundberg-Cooper. These models represent improvements relative to Maxwell inasmuch as these models include 
an anelastic transient regime that allows for generating significant dissipation in the main tidal period range.

Of the other models investigated, all converged upon the same results in terms of interior structure parameters, i.e., the results are to first order insensitive of the exact nature of the attenuation mechanisms that account for dissipation of energy in planetary interiors. While we only examined a single frequency associated with the main tide of Phobos, our results show that from knowledge of the response at an additional period, significantly improved constraints on interior properties can be derived. InSight observations of tidal phase lags will prove particularly rewarding since these appear to be a much better means of discriminating between different models than either tidal amplitudes or induced surface displacements.

As shown here, application of our method yields a host of quantitative predictions and results. In particular, the method also provides insights into future requirements of, e.g., improvements in experimental data, that will be needed for modeling more complex models. Chief among these are (more discussion is given in Nimmo and Faul [2013] and Khan et al. [2018]): a) extending the forced torsional oscillation experiments to minerals beyond olivine, including compositions that are more Fe-rich and therefore more representative of Martian mantle compositions; b) extending the experimental conditions to longer periods; c) consideration of the effects of hydration and partial melt, which can significantly impact viscosity by lowering it and thereby increase dissipation [Jackson et al., 2004; Karato, 2013; Takei, 2017; Cline II et al., 2018]; and d) including grain-size variation with depth in view of geodynamic models that show evidence for grain growth with depth [e.g., Rozel, 2012], which would tend to lower dissipation, requiring increased dissipation elsewhere.

For community use, we computed and tabulated predicted model responses (Love numbers and attenuation) at a number of distinct periods and spherical harmonic degree for each of the rheological models considered here. Since the amount of energy that is being dissipated in planetary interiors depends on rheology, the latter effectively controls the orbital evolution of binaries such as Mars and Phobos and therefore provides an improved means for e.g., understanding the future demise of Phobos. Penultimately, we should note that while the results of this study are based on Mars, the methodology is generally applicable to other terrestrial planets and exoplanets. 
Ultimately, it is the expectation that InSight, which has been operative on the surface of Mars since the end of November 2018, will enable separate measurements of $k_{2}$, $Q_{2}$, and $\delta_{2}$ (and maybe $k_{3}$ and $\delta_{3}$ ). More specifically, and in addition to the direct measurement of the tidal response by RISE, different schemes have been proposed to employ the SEIS instrument to extract the tidal response from the seismic data, by having the very broad-band seismometer act as a gravimeter to measure Mars's response to tidal forces [Pou et al., 2018].

As a final remark, we would like to note that although we have focused on Mars, the methodology developed here is generally formulated and therefore applicable to other solar and extra-solar system bodies, where tidal constraints are available to determine interior structure and properties. In particular, we envision applying our method to the Moon for which tidal dissipation measurements at several periods are available.

\section{A: Viscoelastic parameters}

Table A.1 compiles the viscoelastic parameter values used throughout this study.

\section{B: Further details about tidal calculations}

To model tidal deformation within the planet, we make use of the quasi-static momentum equation [e.g., Dahlen, 1974; Tromp and Mitrovica, 1999; Al-Attar and Tromp, 2014]

$$
-\nabla \cdot \mathbf{T}+\nabla(\rho \mathbf{u} \cdot \nabla \Phi)-\nabla \cdot(\rho \mathbf{u}) \nabla \Phi+\rho \nabla(\phi+\psi)=\mathbf{0},
$$

where $\mathbf{T}$ denotes the incremental Lagrangian-Cauchy stress tensor, $\rho$ the equilibrium density, $\mathbf{u}$ the displacement vector, $\Phi$ the equilibrium gravitational potential, $\phi$ the perturbed gravitational potential, and $\psi$ is the tidal potential that we have now added into the problem. The sign conventions used in this section follow those in Al-Attar and Tromp [2014]. The tidal potential is assumed to have an exponential time-dependence at a given forcing frequency. Due to the linearity of the equations of motion, the displacement and gravitational potential have the time-dependence, and the common exponential factors have been canceled from all equations. The frequency-dependence within the problem then arises solely from the fact that the appropriate viscoelastic modulii are evaluated at the prescribed tidal frequency.

As shown by Dahlen [1974], for static or quasi-static problems this linearised Lagrangian description is only valid within solid parts of the Earth model. Within the fluid 
core, the displacement vector is not well-defined, and Dahlen [1974] instead showed that all relevant fields can be expressed in terms of the perturbed gravitational potential $\phi$. In particular, we can write the first-order perturbations to density $\rho^{\prime}$ and pressure $p^{\prime}$ in the fluid core as

$$
p^{\prime}=-\rho(\phi+\psi), \quad \rho^{\prime}=g^{-1} \partial_{r} \rho(\phi+\psi),
$$

where $g=\partial_{r} \Phi$. These identities generalise those presented in Dahlen [1974] to include the applied tidal potential, but their derivation is essentially unchanged. The gravitational potential perturbation itself is then a solution of the following modified Poisson equation

$$
(4 \pi G)^{-1} \nabla^{2} \phi=\left\{\begin{array}{cc}
-\nabla \cdot(\rho \mathbf{u}) & \text { in solid regions } \\
g^{-1} \partial_{r} \rho(\phi+\psi) & \text { in fluid regions } \\
0 & \text { outside the planet }
\end{array}\right.
$$

where $G$ is Newton's gravitational constant. The boundary and continuity conditions for the problem can be found in detail in Al-Attar and Tromp [2014]. Within the tidal problem, however, there is no applied surface load, while the tidal potential $\psi$ appears within the continuity conditions on the linearised traction across fluid-solid boundaries via its occurrence in the pressure perturbation $p^{\prime}$ in fluid regions.

For numerical work, it is most convenient to express the problem in its weak form. The derivation follows closely that given in Al-Attar and Tromp [2014], requiring only slight changes due to the inclusion of the tidal potential in the momentum equation, the modified Possion equation, and in the traction boundary conditions at fluid-solid boundaries. The final result is given by

$$
\begin{aligned}
\mathcal{A}\left(\mathbf{u}, \phi \mid \mathbf{u}^{\prime}, \phi^{\prime}\right) & +\int_{M_{S}} \rho \nabla \psi \cdot \mathbf{u}^{\prime} \mathrm{d} V+\int_{M_{F}} g^{-1} \partial_{r} \rho \psi \phi^{\prime} \mathrm{d} V \\
& +\int_{\Sigma_{F S}} \rho^{-} \psi \mathbf{u}^{\prime} \cdot \hat{\mathbf{n}} \mathrm{d} S-\int_{\Sigma_{S F}} \rho^{+} \psi \mathbf{u}^{\prime} \cdot \hat{\mathbf{n}} \mathrm{d} S=0,
\end{aligned}
$$

where $\mathcal{A}$ is the bilinear form defined in eq.(2.52) of Al-Attar and Tromp [2014], $\left(\mathbf{u}^{\prime}, \phi^{\prime}\right)$ are test functions for the displacement and potential, respectively, $M_{S}$ denotes the solid regions of the model, $M_{F}$ the fluid regions, $\Sigma_{F S}$ and $\Sigma_{S F}$ denote the fluid-solid boundaries, where the first subscript indicates whether the region on the inside of the boundary is solid (S) or fluid (F), and finally $\rho^{-}$and $\rho^{+}$denote, respectively the equilibrium density evaluated on the lower or upper sides of a boundary. As the tidal potential only modifies the force term for the problem, the numerical implementation was readily made within the loading code developed by Al-Attar and Tromp [2014], which has been subsequently refined and improved by Crawford et al. [2018]. 


\section{C: Seismic wave speed and density profiles}

Figure C.1 shows sampled $P$ - and $S$-wave speed and density profiles from the surface to the centre of Mars for each of the rheological models considered here. As the figures also shows, the particular choice of rheological model does not appear to make a substantial difference, since the solutions for the various viscoelastic models largely overlap. In the context of investigating the influence of compositional variations, [Khan et al., 2018] examined four other bulk Martian compositions (Sanloup et al. [1999], Lodders and Fegley [1997], Dreibus and Wänke [1984], Morgan and Anders [1979]) that resulted in models that are consistent with the present results (see also section 5.3).

\section{Acknowledgments}

We are grateful to two anynomous reviewers for comments that improved the manuscript. We would also like to thank Michael Efroimsky and Francis Nimmo for additional suggestions. This work was supported by a grant from the Swiss National Science Foundation (SNF project 172508 "Mapping the internal structure of Mars"). This is InSight contribution number 92. The interior structure models computed here, including inverted model parameters, are available online at: https://github.com/bagheriamirh/Tidal-Response-ofMars-JGR.

\section{References}

A, G., J. Wahr, and S. Zhong (2014), The effects of laterally varying icy shell structure on the tidal response of Ganymede and Europa, Journal of Geophysical Research (Planets), 119, 659-678, doi:10.1002/2013JE004570.

Abers, G. A., K. Fischer, G. Hirth, D. Wiens, T. Plank, B. K. Holtzman, C. McCarthy, and E. Gazel (2014), Reconciling mantle attenuation-temperature relationships from seismology, petrology, and laboratory measurements, Geochemistry, Geophysics, Geosystems, 15(9), 3521-3542.

Agnew, D. (2015), Earth Tides. Treatise on Geophysics and Geodesy, 151-178 pp., New York: Elsevier.

Al-Attar, D., and J. Tromp (2014), Sensitivity kernels for viscoelastic loading based on adjoint methods, Geophysical Journal International, 196(1), 34-77, doi: 10.1093/gji/ggt395. 
Andrade, E. (1962), The validity of the t1/3 law of flow of metals, Philosophical Magazine, 7(84), 2003-2014.

Bellis, C., and B. Holtzman (2014), Sensitivity of seismic measurements to frequencydependent attenuation and upper mantle structure: An initial approach, Journal of Geophysical Research: Solid Earth, 119(7), 5497-5517, doi:10.1002/2013JB010831.

Benjamin, D., J. Wahr, R. D. Ray, G. D. Egbert, and S. D. Desai (2006), Constraints on mantle anelasticity from geodetic observations, and implications for the $\mathbf{J}_{2}$ anomaly, Geophysical Journal International, 165, 3-16, doi:10.1111/j.1365-246X.2006.02915.x.

Bierson, C. J., and F. Nimmo (2016), A test for io's magma ocean: Modeling tidal dissipation with a partially molten mantle, Journal of Geophysical Research: Planets, 121(11), 2211-2224, doi:10.1002/2016JE005005.

Bills, B. G., G. A. Neumann, D. E. Smith, and M. T. Zuber (2005), Improved estimate of tidal dissipation within Mars from MOLA observations of the shadow of Phobos, Journal of Geophysical Research (Planets), 110, E07004, doi:10.1029/2004JE002376.

Black, B. A., and T. Mittal (2015), The demise of phobos and development of a martian ring system, Nature Geoscience, 8(12), 913.

Breuer, D., D. A. Yuen, and T. Spohn (1997), Phase transitions in the Martian mantle: Implications for partially layered convection, Earth and Planetary Science Letters, 148, 457-469, doi:10.1016/S0012-821X(97)00049-6.

Burns, J. A. (1978), The dynamical evolution and origin of the martian moons, Vistas in Astronomy, 22, 193-210.

Castillo-Rogez, J. C., and W. B. Banerdt (2012), Impact of Anelasticity on Mars' Dissipative Properties - Application to the InSight Mission, in The Mantle of Mars: Insights from Theory, Geophysics, High-Pressure Studies, and Meteorites, LPI Contributions, vol. 1684, p. 4.

Castillo-Rogez, J. C., M. Efroimsky, and V. Lainey (2011), The tidal history of Iapetus: Spin dynamics in the light of a refined dissipation model, Journal of Geophysical Research (Planets), 116, E09008, doi:10.1029/2010JE003664.

Cathles, L. M. (2015), Viscosity of the Earth's Mantle, vol. 1362, Princeton University Press.

Chawla, K. K., and M. Meyers (1999), Mechanical behavior of materials, Prentice Hall Upper Saddle River. 
Choblet, G., G. Tobie, C. Sotin, M. Běhounková, O. Čadek, F. Postberg, and O. Souček (2017), Powering prolonged hydrothermal activity inside enceladus, Nature Astronomy, $1(12), 841$.

Cline II, C., U. Faul, E. David, A. Berry, and I. Jackson (2018), Redox-influenced seismic properties of upper-mantle olivine, Nature, 555(7696), 355.

Connolly, J. A. D. (2009), The geodynamic equation of state: What and how, Geochemistry, Geophysics, Geosystems, 10(10), n/a-n/a, doi:10.1029/2009GC002540, q10014.

Cooper, R. F. (2002), Seismic wave attenuation: Energy dissipation in viscoelastic crystalline solids, Reviews in mineralogy and geochemistry, 51(1), 253-290.

Correia, A. C., G. Boué, J. Laskar, and A. Rodríguez (2014), Deformation and tidal evolution of close-in planets and satellites using a maxwell viscoelastic rheology, Astronomy \& Astrophysics, 571, A50.

Crawford, O., D. Al-Attar, J. Tromp, J. X. Mitrovica, J. Austermann, and H. C. P. Lau (2018), Quantifying the sensitivity of post-glacial sea level change to laterally varying viscosity, Geophysical Journal International, 214(2), 1324-1363, doi: $10.1093 / \mathrm{gji} / \mathrm{ggy} 184$.

Dahlen, F. A. (1974), On the Static Deformation of an Earth Model with a Fluid Core, Geophysical Journal, 36, 461-485, doi:10.1111/j.1365-246X.1974.tb03649.x.

Dreibus, G., and H. Wänke (1984), Accretion of the earth and inner planets., in Geochemistry and Cosmochemistry, pp. 3-11.

Dumoulin, C., G. Tobie, O. Verhoeven, P. Rosenblatt, and N. Rambaux (2017), Tidal constraints on the interior of venus, Journal of Geophysical Research: Planets, 122(6), $1338-1352$.

Efroimsky, M. (2012a), Tidal Dissipation Compared to Seismic Dissipation: In Small Bodies, Earths, and Super-Earths, Astrophys. J., 746, 150, doi:10.1088/0004$637 \mathrm{X} / 746 / 2 / 150$.

Efroimsky, M. (2012b), Bodily tides near spin-orbit resonances, Celestial Mechanics and Dynamical Astronomy, 112(3), 283-330, doi:10.1007/s10569-011-9397-4.

Efroimsky, M. (2015), Tidal evolution of asteroidal binaries. ruled by viscosity. ignorant of rigidity, The Astronomical Journal, 150(4), 98.

Efroimsky, M., and V. Lainey (2007), Physics of bodily tides in terrestrial planets and the appropriate scales of dynamical evolution, Journal of Geophysical Research (Planets), 112(E11), E12003, doi:10.1029/2007JE002908. 
Efroimsky, M., and V. V. Makarov (2013), Tidal Friction and Tidal Lagging. Applicability Limitations of a Popular Formula for the Tidal Torque, apj, 764, 26, doi:10.1088/0004$637 \mathrm{X} / 764 / 1 / 26$.

Efroimsky, M., and V. V. Makarov (2014), Tidal dissipation in a homogeneous spherical body. i. methods, The Astrophysical Journal, 795(1), 6.

Faul, U., and I. Jackson (2015), Transient creep and strain energy dissipation: An experimental perspective, Annual Review of Earth and Planetary Sciences, 43, 541-569, doi: 10.1146/annurev-earth-060313-054732.

Findley, L., and K. Onaran (1965), Creep and Relaxation of Nonlinear Viscoelastic Materials, Dover Publications, New York.

Folkner, W. M., V. Dehant, S. Le Maistre, M. Yseboodt, A. Rivoldini, T. Van Hoolst, S. W. Asmar, and M. P. Golombek (2018), The rotation and interior structure experiment on the insight mission to mars, Space Science Reviews, 214(5), 100.

Forte, A. M., and J. X. Mitrovica (2001), Deep-mantle high-viscosity flow and thermochemical structure inferred from seismic and geodynamic data, nature, 410, 1049-1056.

Genova, A., S. Goossens, F. G. Lemoine, E. Mazarico, G. A. Neumann, D. E. Smith, and M. T. Zuber (2016), Seasonal and static gravity field of Mars from MGS, Mars Odyssey and MRO radio science, icarus, 272, 228-245, doi:10.1016/j.icarus.2016.02.050.

Gribb, T. T., and R. F. Cooper (1998), Low-frequency shear attenuation in polycrystalline olivine: Grain boundary diffusion and the physical significance of the andrade model for viscoelastic rheology, Journal of Geophysical Research: Solid Earth, 103(B11), $27,267-27,279$.

Harada, Y., S. Goossens, K. Matsumoto, J. Yan, J. Ping, H. Noda, and J. Haruyama (2014), Strong tidal heating in an ultralow-viscosity zone at the core-mantle boundary of the Moon, Nature Geoscience, 7, 569-572, doi:10.1038/ngeo2211.

Hauck, S. A., J.-L. Margot, S. C. Solomon, R. J. Phillips, C. L. Johnson, F. G. Lemoine, E. Mazarico, T. J. McCoy, S. Padovan, S. J. Peale, et al. (2013), The curious case of mercury's internal structure, Journal of Geophysical Research: Planets, 118(6), 12041220.

Helffrich, G. (2017), Mars core structure-concise review and anticipated insights from insight, Progress in Earth and Planetary Science, 4(1), 24, doi:10.1186/s40645-017$0139-4$ 
Henning, W. G., R. J. O’Connell, and D. D. Sasselov (2009), Tidally heated terrestrial exoplanets: viscoelastic response models, The Astrophysical Journal, 707(2), 1000.

Hussmann, H., and T. Spohn (2004), Thermal-orbital evolution of io and europa, Icarus, 171(2), 391 - 410, doi:https://doi.org/10.1016/j.icarus.2004.05.020.

Jackson, I., and U. H. Faul (2010), Grainsize-sensitive viscoelastic relaxation in olivine: Towards a robust laboratory-based model for seismological application, Physics of the Earth and Planetary Interiors, 183, 151-163, doi:10.1016/j.pepi.2010.09.005.

Jackson, I., J. D. Fitz Gerald, U. H. Faul, and B. H. Tan (2002), Grain-size-sensitive seismic wave attenuation in polycrystalline olivine, Journal of Geophysical Research: Solid Earth, 107(B12), ECV 5-1-ECV 5-16, doi:10.1029/2001JB001225, 2360.

Jackson, I., U. H. Faul, J. D. F. Gerald, and B. H. Tan (2004), Shear wave attenuation and dispersion in melt-bearing olivine polycrystals: 1 . specimen fabrication and mechanical testing, Journal of Geophysical Research: Solid Earth, 109(B6).

Jackson, I., et al. (2007), Properties of rock and minerals-physical origins of anelasticity and attenuation in rock, in Treatise on geophysics, Elsevier.

Kamata, S., J. Kimura, K. Matsumoto, F. Nimmo, K. Kuramoto, and N. Namiki (2016), Tidal deformation of Ganymede: Sensitivity of Love numbers on the interior structure, Journal of Geophysical Research (Planets), 121, 1362-1375, doi:10.1002/2016JE005071.

Karato, S., and H. A. Spetzler (1990), Defect microdynamics in minerals and solid-state mechanisms of seismic wave attenuation and velocity dispersion in the mantle, Reviews of Geophysics, 28(4), 399-421, doi:10.1029/RG028i004p00399.

Karato, S.-I. (1984), Grain-size distribution and rheology of the upper mantle, Tectonophysics, 104(1-2), 155-176.

Karato, S. I. (2008), Deformation of Earth Materials, Cambridge University Press.

Karato, S.-i. (2013), Geophysical constraints on the water content of the lunar mantle and its implications for the origin of the Moon, Earth and Planetary Science Letters, 384, 144-153, doi:10.1016/j.epsl.2013.10.001.

Karato, S.-i., T. Olugboji, and J. Park (2015), Mechanisms and geologic significance of the mid-lithosphere discontinuity in the continents, Nature geoscience, 8(7), 509.

Khan, A., and J. A. D. Connolly (2008), Constraining the composition and thermal state of Mars from inversion of geophysical data, Journal of Geophysical Research (Planets), 113, E07003, doi:10.1029/2007JE002996. 
Khan, A., C. Liebske, A. Rozel, A. Rivoldini, F. Nimmo, J. A. D. Connolly, A. Plesa, and D. Giardini (2018), A geophysical perspective on the bulk composition of mars, Journal of Geophysical Research: Planets, 123(2), 575-611, doi:10.1002/2017JE005371.

Konopliv, A. S., and C. F. Yoder (1996), Venusian k2 tidal love number from magellan and pvo tracking data, Geophysical Research Letters, 23(14), 1857-1860, doi: 10.1029/96GL01589.

Konopliv, A. S., R. S. Park, and W. M. Folkner (2016), An improved JPL Mars gravity field and orientation from Mars orbiter and lander tracking data, icarus, 274, 253-260, doi:10.1016/j.icarus.2016.02.052.

Lainey, V., V. Dehant, and M. Pätzold (2007), First numerical ephemerides of the Martian moons, Astronomy and Astrophysics, 465, 1075-1084, doi:10.1051/0004-6361:20065466.

Lau, H. C., and U. H. Faul (2019), Anelasticity from seismic to tidal timescales: Theory and observations, Earth and Planetary Science Letters, 508, 18-29.

Lau, H. C., U. Faul, J. X. Mitrovica, D. Al-Attar, J. Tromp, and G. GarapiäĞ (2016), Anelasticity across seismic to tidal timescales: a self-consistent approach, Geophysical Journal International, 208(1), 368-384, doi:10.1093/gji/ggw401.

Lodders, K., and B. Fegley (1997), An Oxygen Isotope Model for the Composition of Mars, icarus, 126, 373-394, doi:10.1006/icar.1996.5653.

Lognonné, P., and B. Mosser (1993), Planetary seismology, Surveys in Geophysics, 14, 239-302, doi:10.1007/BF00690946.

Lognonné, P., J. G. Beyneix, W. B. Banerdt, S. Cacho, J. F. Karczewski, and M. Morand (1996), Ultra broad band seismology on InterMarsNet, Planets and Space Science, 44, 1237, doi:10.1016/S0032-0633(96)00083-9.

Lognonné, P. e. a. (2019), Seis: Insight's seismic experiment for internal structure of mars, Space Science Reviews, 215(1), 12, doi:10.1007/s11214-018-0574-6.

McCarthy, C., and J. C. Castillo-Rogez (2013), Planetary ices attenuation properties, in The Science of Solar System Ices, pp. 183-225, Springer.

McCarthy, C., Y. Takei, and T. Hiraga (2011), Experimental study of attenuation and dispersion over a broad frequency range: 2 . the universal scaling of polycrystalline materials, Journal of Geophysical Research: Solid Earth, 116(B9), n/a-n/a, doi: 10.1029/2011JB008384, b09207.

McSween, H. Y., Jr., and S. M. McLennan (2014), Mars, in Planets, Asteriods, Comets and The Solar System, edited by K. Turekian and H. Holland, pp. 251-300, Elsevier Science, 
doi:10.1016/B978-0-08-095975-7.00125-X.

Minster, J. B., and D. L. Anderson (1981), A model of dislocation-controlled rheology for the mantle, The Royal Society, 299(1449), 319-356, doi:10.1098/rsta.1981.0025.

Moczo, P., and J. Kristek (2005), On the rheological models used for time-domain methods of seismic wave propagation, Geophysical Research Letters, 32(1), doi: 10.1029/2004GL021598.

Moore, W. B., and G. Schubert (2000), NOTE: The Tidal Response of Europa, icarus, 147, 317-319, doi:10.1006/icar.2000.6460.

Morgan, J. W., and E. Anders (1979), Mars: a cosmochemical-geophysical estimate of bulk composition, in Mars, p. 60.

Morris, S., and I. Jackson (2009), Implications of the similarity principle relating creep and attenuation in finely grained solids, Materials Science and Engineering: A, 521, $124-127$.

Mosegaard, K., and A. Tarantola (1995), Monte carlo sampling of solutions to inverse problems, Journal of Geophysical Research: Solid Earth(1978-2012), 100(B7), 12,43112,447 .

Nimmo, F., and U. Faul (2013), Dissipation at tidal and seismic frequencies in a melt-free, anhydrous mars, Journal of Geophysical Research: Planets, 118(12), 2558-2569.

Nimmo, F., U. H. Faul, and E. J. Garnero (2012), Dissipation at tidal and seismic frequencies in a melt-free Moon, Journal of Geophysical Research (Planets), 117, E09005, doi: 10.1029/2012JE004160.

Nowick, A. S., and B. Berry (1972), Anelastic relaxation in crystalline solids, Academic Press.

Padovan, S., J. Margot, S. A. Hauck, W. B. Moore, and S. C. Solomon (2013), The tides of mercury and possible implications for its interior structure, Journal of Geophysical Research: Planets, 119(4), 850-866, doi:10.1002/2013JE004459.

Peale, S. (1977), Rotation histories of the natural satellites, in IAU Colloq. 28: Planetary Satellites, pp. 87-111.

Peltier, W. (1974), The impulse response of a maxwell earth, Reviews of Geophysics, 12(4), 649-669.

Plesa, A.-C., M. Grott, N. Tosi, D. Breuer, T. Spohn, and M. A. Wieczorek (2016), How large are present-day heat flux variations across the surface of Mars?, Journal of Geophysical Research (Planets), 121, 2386-2403, doi:10.1002/2016JE005126. 
Pou, L., D. Mimoun, P. Lognonne, R. F. Garcia, O. Karatekin, M. Nonon-Latapie, and R. Llorca-Cejudo (2018), High precision seis calibration for the insight mission and its applications, Space Science Reviews, 215(1), 6, doi:10.1007/s11214-018-0561-y.

Qin, C., S. Zhong, and J. Wahr (2016), Elastic tidal response of a laterally heterogeneous planet: a complete perturbation formulation, Geophysical Journal International, 207(1), $89-110$

Raj, R., and M. Ashby (1975), Intergranular fracture at elevated temperature, Acta Metallurgica, 23(6), 653-666.

Ranalli, G. (2001), Mantle rheology: radial and lateral viscosity variations inferred from microphysical creep laws, Journal of Geodynamics, 32(4), 425 - 444, doi: https://doi.org/10.1016/S0264-3707(01)00042-4.

Remus, F., S. Mathis, J.-P. Zahn, and V. Lainey (2012), Anelastic tidal dissipation in multi-layer planets, Astronomy \& Astrophysics, 541, A165.

Renaud, J. P., and W. G. Henning (2018), Increased tidal dissipation using advanced rheological models: Implications for io and tidally active exoplanets, The Astrophysical Journal, 857(2), 98 .

Rivoldini, A., T. Van Hoolst, O. Verhoeven, A. Mocquet, and V. Dehant (2011), Geodesy constraints on the interior structure and composition of Mars, Icarus, 213, 451-472, doi:10.1016/j.icarus.2011.03.024.

Roberts, J. H., and F. Nimmo (2008), Tidal heating and the long-term stability of a subsurface ocean on Enceladus, icarus, 194, 675-689, doi:10.1016/j.icarus.2007.11.010.

Robuchon, G., G. Choblet, G. Tobie, O. Čadek, C. Sotin, and O. Grasset (2010), Coupling of thermal evolution and despinning of early iapetus, Icarus, 207(2), 959-971.

Rosenblatt, P., S. Charnoz, K. M. Dunseath, M. Terao-Dunseath, A. Trinh, R. Hyodo, H. Genda, and S. Toupin (2016), Accretion of phobos and deimos in an extended debris disc stirred by transient moons, Nature Geoscience, 9(8), 581.

Rozel, A. (2012), Impact of grain size on the convection of terrestrial planets, Geochemistry, Geophysics, Geosystems, 13(10), doi:10.1029/2012GC004282.

Ruedas, T., P. J. Tackley, and S. C. Solomon (2013), Thermal and compositional evolution of the martian mantle: Effects of phase transitions and melting, Physics of the Earth and Planetary Interiors, 216, 32-58, doi:10.1016/j.pepi.2012.12.002.

Sanloup, C., A. Jambon, and P. Gillet (1999), A simple chondritic model of Mars, Physics of the Earth and Planetary Interiors, 112, 43-54, doi:10.1016/S0031-9201(98)00175-7. 
Sasaki, Y., Y. Takei, C. McCarthy, and J. F. Rudge (2019), Experimental study of dislocation damping using a rock analogue, Journal of Geophysical Research: Solid Earth.

Seidelmann, P. K., V. K. Abalakin, M. Bursa, M. E. Davies, C. de Bergh, J. H. Lieske, J. Oberst, J. L. Simon, E. M. Standish, P. Stooke, and P. C. Thomas (2002), Report of the iau/iag working group on cartographic coordinates and rotational elements of the planets and satellites: 2000, Celestial Mechanics and Dynamical Astronomy, 82(1), 83111, doi:10.1023/A:1013939327465.

Smrekar, S. E., P. Lognonné, T. Spohn, W. B. Banerdt, D. Breuer, U. Christensen, V. Dehant, M. Drilleau, W. Folkner, N. Fuji, et al. (2019), Pre-mission insights on the interior of mars, Space Science Reviews, 215(1), 3.

Sohl, F., G. Schubert, and T. Spohn (2005), Geophysical constraints on the composition and structure of the Martian interior, Journal of Geophysical Research (Planets), 110(E9), E12008, doi:10.1029/2005JE002520.

Soldati, G., L. Boschi, F. Deschamps, and D. Giardini (2009), Inferring radial models of mantle viscosity from gravity (GRACE) data and an evolutionary algorithm, Physics of the Earth and Planetary Interiors, 176, 19-32, doi:10.1016/j.pepi.2009.03.013.

Stevenson, D. J. (2001), Mars' core and magnetism, nature, 412, 214-219.

Stewart, A. J., M. W. Schmidt, W. van Westrenen, and C. Liebske (2007), Mars: A New Core-Crystallization Regime, Science, 316, 1323, doi:10.1126/science.1140549.

Stixrude, L., and C. Lithgow-Bertelloni (2005b), Mineralogy and elasticity of the oceanic upper mantle: Origin of the low-velocity zone, Journal of Geophysical Research: Solid Earth, 110(B3), doi:10.1029/2004JB002965, b03204.

Stixrude, L., and C. Lithgow-Bertelloni (2011), Thermodynamics of mantle minerals - II. Phase equilibria, Geophysical Journal International, 184, 1180-1213, doi: 10.1111/j.1365-246X.2010.04890.x.

Sundberg, M., and R. Cooper (2010), A composite viscoelastic model for incorporating grain boundary sliding and transient diffusion creep; correlating creep and attenuation responses for materials with a fine grain size, Philosophical Magazine, 90(20), 28172840, doi:10.1080/14786431003746656.

Takei, Y. (2017), Effects of partial melting on seismic velocity and attenuation: A new insight from experiments, Annual Review of Earth and Planetary Sciences, 45(1), null, doi:10.1146/annurev-earth-063016-015820. 
Takei, Y., F. Karasawa, and H. Yamauchi (2014), Temperature, grain size, and chemical controls on polycrystal anelasticity over a broad frequency range extending into the seismic range, Journal of Geophysical Research: Solid Earth, 119(7), 5414-5443, doi: 10.1002/2014JB011146.

Taylor, G. J. (2013), The bulk composition of mars, Chemie Der Erde-Geochemistry, 73(4), 401-420.

Taylor, S. R., and S. McLennan (2008), Planetary Crusts: Their Composition, Origin and Evolution, Cambridge Planetary Science, Cambridge University Press, doi: 10.1017/CBO9780511575358.

Tromp, J., and J. X. Mitrovica (1999), Surface loading of a viscoelastic earth-I. General theory, Geophysical Journal International, 137, 847-855, doi:10.1046/j.1365246X.1999.00838.x.

Van Hoolst, T., V. Dehant, F. Roosbeek, and P. Lognonné (2003), Tidally induced surface displacements, external potential variations, and gravity variations on Mars, Icarus, 161, 281-296, doi:10.1016/S0019-1035(02)00045-3.

van Thienen, P., A. Rivoldini, T. Van Hoolst, and P. Lognonné (2006), A top-down origin for martian mantle plumes, icarus, 185, 197-210, doi:10.1016/j.icarus.2006.06.008.

Wahr, J., Z. A. Selvans, M. E. Mullen, A. C. Barr, G. C. Collins, M. M. Selvans, and R. T. Pappalardo (2009), Modeling stresses on satellites due to nonsynchronous rotation and orbital eccentricity using gravitational potential theory, icarus, 200, 188-206, doi:10.1016/j.icarus.2008.11.002.

Williams, J. G., and D. H. Boggs (2015), Tides on the Moon: Theory and determination of dissipation, Journal of Geophysical Research (Planets), 120, 689-724, doi: 10.1002/2014JE004755.

Williams, J. G., S. G. Turyshev, D. H. Boggs, and J. T. Ratcliff (2006), Lunar laser ranging science: gravitational physics and lunar interior and geodesy, Advances in Space Research, 37(1), 67-71.

Williams, J. G., A. S. Konopliv, D. H. Boggs, R. S. Park, D.-N. Yuan, F. G. Lemoine, S. Goossens, E. Mazarico, F. Nimmo, R. C. Weber, S. W. Asmar, H. J. Melosh, G. A. Neumann, R. J. Phillips, D. E. Smith, S. C. Solomon, M. M. Watkins, M. A. Wieczorek, J. C. Andrews-Hanna, J. W. Head, W. S. Kiefer, I. Matsuyama, P. J. McGovern, G. J. Taylor, and M. T. Zuber (2014), Lunar interior properties from the GRAIL mission, Journal of Geophysical Research (Planets), 119, 1546-1578, doi: 
10.1002/2013JE004559.

Yoder, C. F. (1995), Venus' free obliquity, Icarus, 117(2), 250-286.

Yoder, C. F., A. S. Konopliv, D. N. Yuan, E. M. Standish, and W. M. Folkner (2003), Fluid Core Size of Mars from Detection of the Solar Tide, Science, 300, 299-303, doi: 10.1126/science.1079645.

Zharkov, V. N., and T. V. Gudkova (1997), On the dissipative factor of the Martian interiors, Planets and Space Science, 45, 401-407, doi:10.1016/S0032-0633(96)00144-4.

Zharkov, V. N., and T. V. Gudkova (2005), Construction of Martian Interior Model, Solar System Research, 39, 343-373, doi:10.1007/s11208-005-0049-7.

Zharkov, V. N., and T. V. Gudkova (2009), The period and Q of the Chandler wobble of Mars, planss, 57, 288-295, doi:10.1016/j.pss.2008.11.010. 

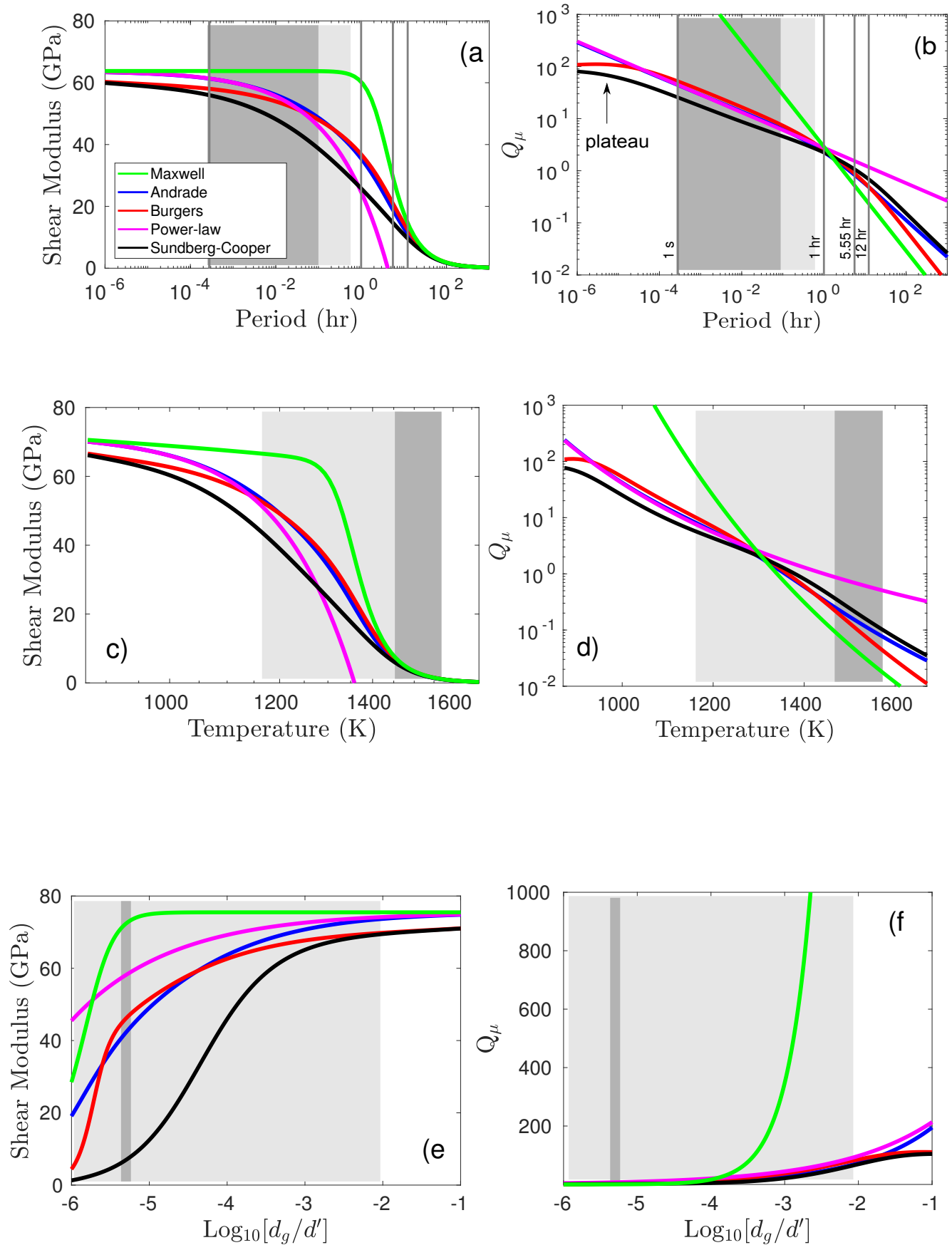

Figure 3. Computed variations of relaxed shear modulus $\left(G_{R}\right)$ and shear attenuation $\left(Q_{\mu}\right)$ with period, temperature, and grain size for the five rheological models considered in this study. (a, b) $G_{R}$ and $Q_{\mu}$ as a function of period at constant temperature and grain size, The vertical lines show periods of interest: seismic body waves ( $1 \mathrm{~s})$, normal modes (1 hr), main tidal excitation of Phobos (5.55 hr), and main tidal excitation of the Sun (12.32 hr). (c, d) $G_{R}$ and $Q_{\mu}$ as a function of temperature at constant period and grain size, $(\mathrm{e}, \mathrm{f})$ $G_{R}$ and $Q_{\mu}$ as a function of grain size at constant period and temperature. Light and dark shaded areas denote the ranges covered by the experimental measurements of Jackson and Faul [2010] and Sundberg and Cooper [2010], respectively. All curves were produced at a constant pressure of $10.4 \mathrm{GPa}$ and for an unrelaxed shear $-44$ modulus of $65 \mathrm{GPa}$. Viscoelastic parameter values employed are given in Table A.1 and $d^{\prime}=1 \mathrm{~m}$. 


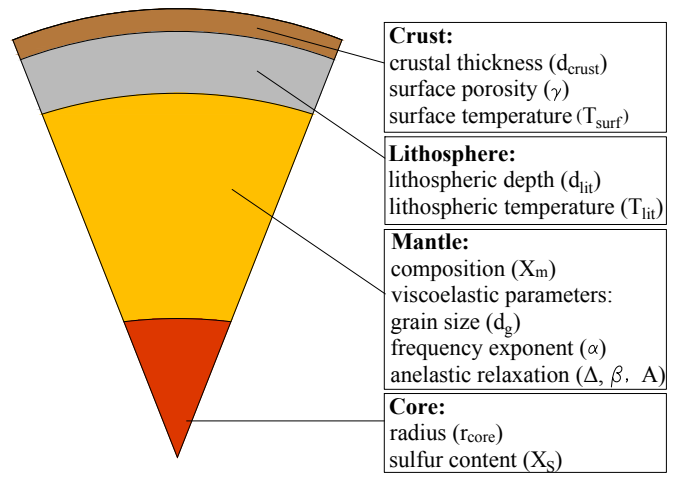

Figure 4. Schematic diagram illustrating model parameterization. The model is spherically symmetric and divided into crust, lithosphere, mantle, and core. These fours layers are parameterized using the parameters shown in the boxes on the right. For more details see main text (section 4.1).
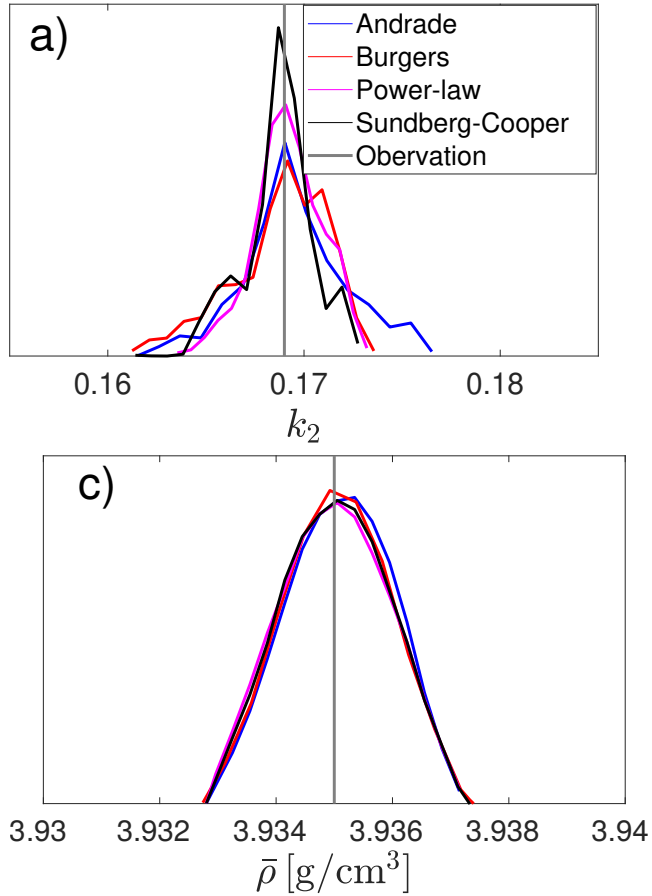
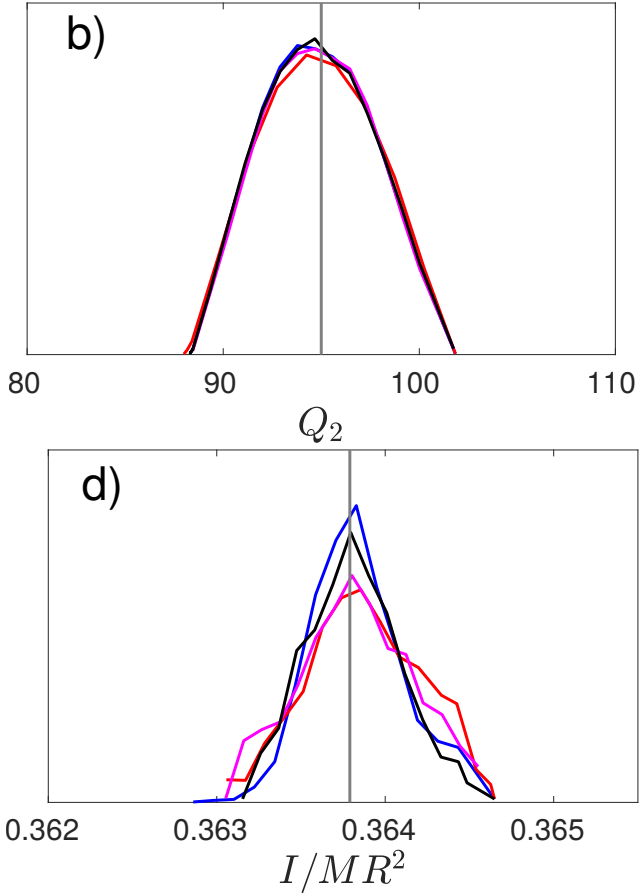

Figure 5. Computed data distributions showing fit to observations for each of the rheological models: a) second-degree tidal Love number $k_{2}$; b) second-degree global tidal dissipation $Q_{2} ;$ c) mean density $\bar{\rho}$; and d) mean moment of inertia $I / M R^{2}$. The results shown in (a) and (b) refer to the main tidal period of Phobos. The vertical solid lines indicate observed values of $k_{2}, Q_{2}, \bar{\rho}$, and $I / M R^{2}$. Observations and uncertainties are compiled in table 1 . 


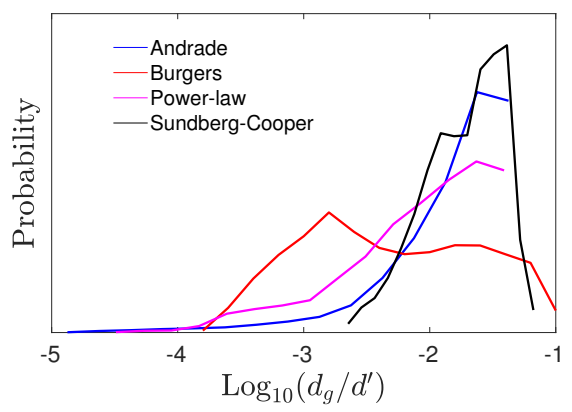

Figure 6. Sampled distributions of grain-size for each viscoelastic model obtained from the inversion.
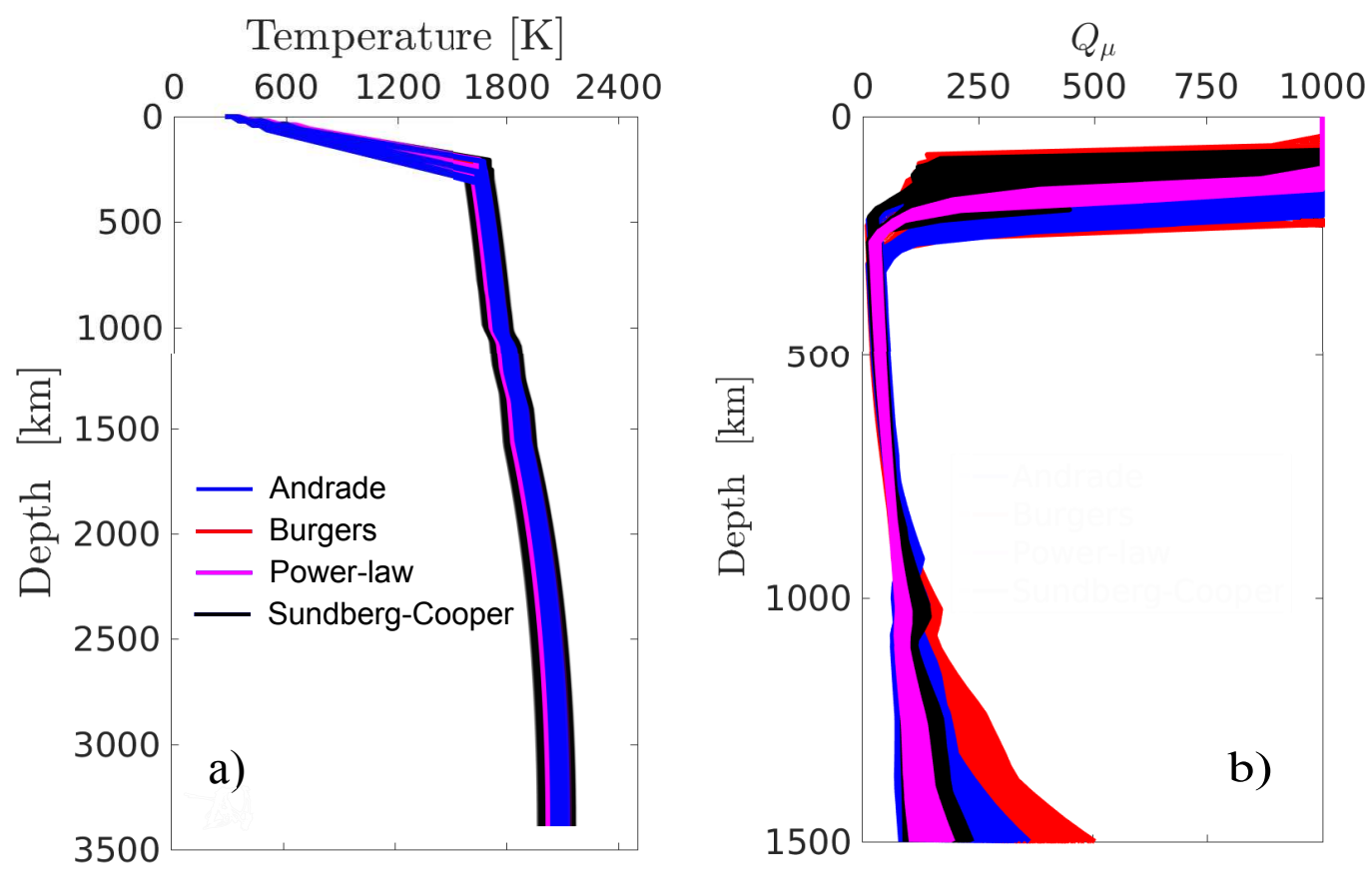

Figure 7. Inverted areothermal (a) and shear attenuation (b) profiles for the main viscoelastic models considered in this study (at the main tidal period of Phobos). Shear attenuation models are only shown down to the core-mantle-boundary since the core is fluid $\left(Q_{\mu}=0\right)$. 


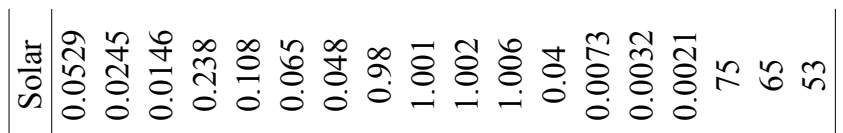

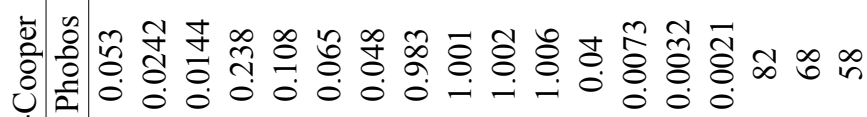

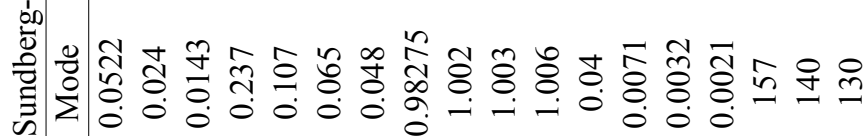

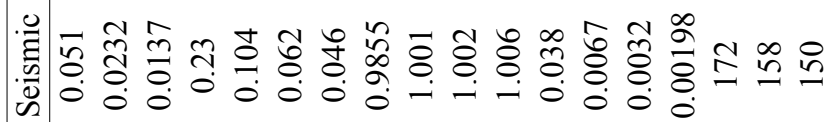

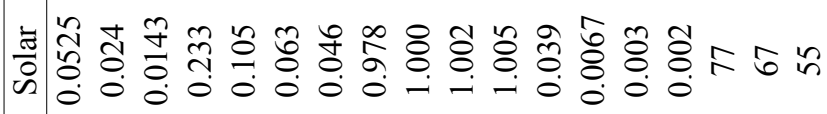

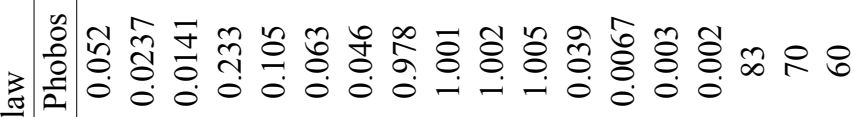
ग)

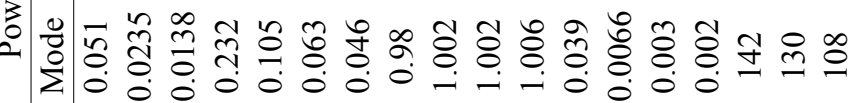

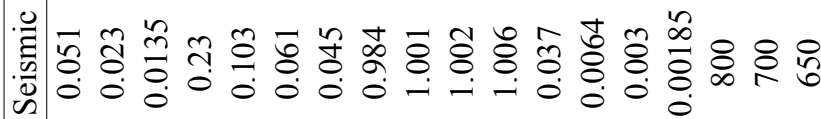

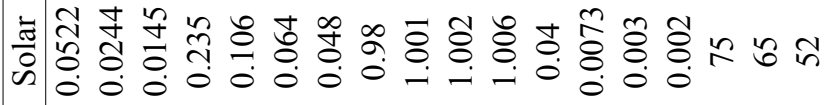

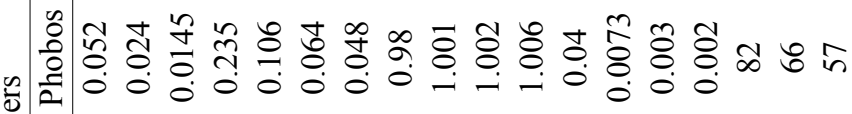
营

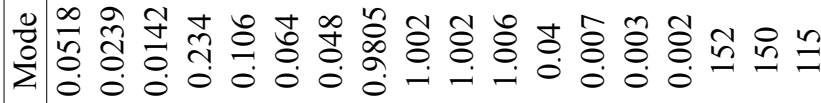

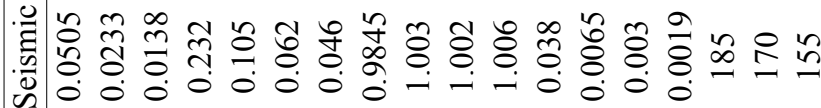

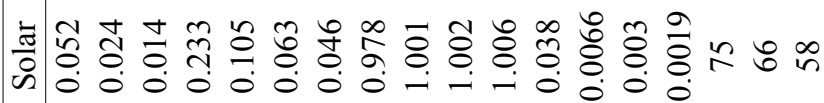

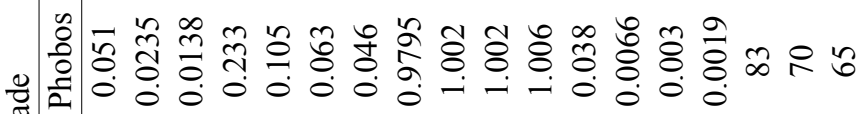
突

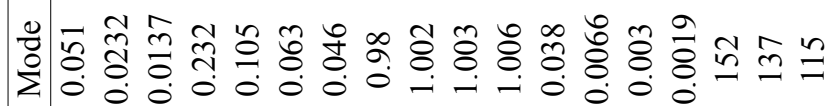

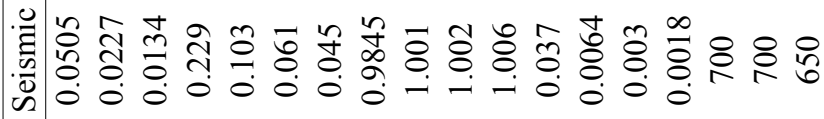

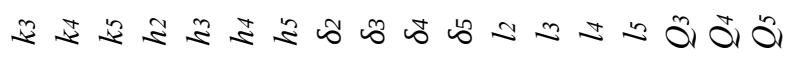



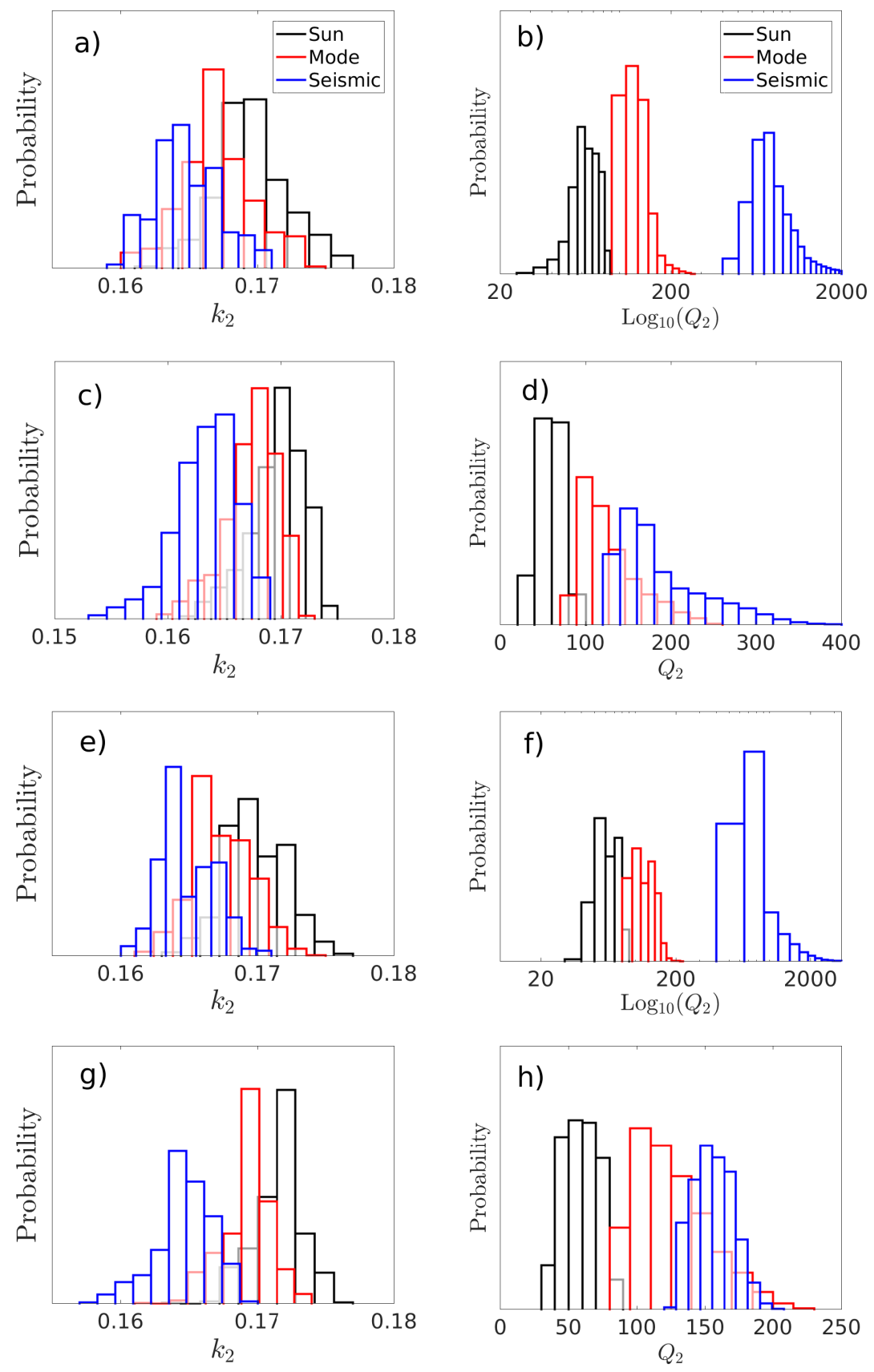

Figure 8. Sampled distributions of second-degree tidal Love number $k_{2}$ and quality factor $Q_{2}$ at three different periods of geophysical interest for each rheological model: a-b) Andrade, c-d) extended Burgers, e-f) power-law, and g-h) Sundberg-Cooper. Note that because of the large variation in $Q_{2}$ for the Andrade and power-law models, plots b) and f) are shown in terms of $\log _{10}\left(Q_{2}\right)$. The distributions represent predictions based on the observed 5.55-hr main Phobos tide. The periods considered are: $12.32 \mathrm{hr}$ (solar tide), $1 \mathrm{hr}$ (long-period normal modes), and $1 \mathrm{~s}$ (short-period body waves). 

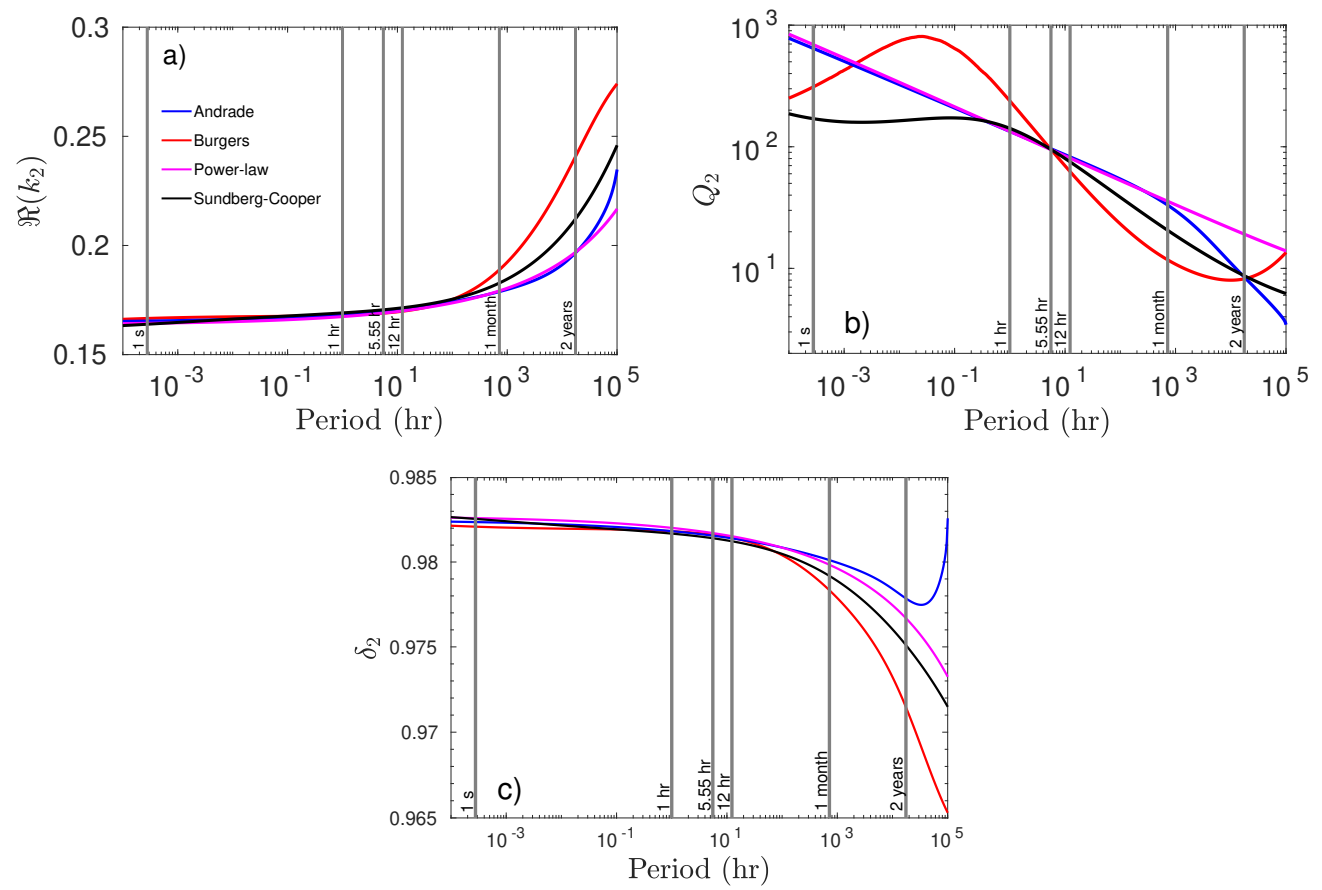

Figure 9. Computed tidal response of Mars as a function of period from short-period seismic ( $1 \mathrm{~s})$ to longperiod tidal time scales ( $\sim 10 \mathrm{yr})$ for the four major rheological models considered in this study. a) Amplitude of tidal response (real part of second-degree potential Love number $k_{2}$ ), b) second-degree global tidal quality factor $\left(Q_{2}\right)$, and c) gravimetric factor $\left(\delta_{2}\right)$. The response curves were computed using the maximum likelihood model obtained in the inversion and the viscoelastic parameters compiled in the Table A.1 for each rheology. 


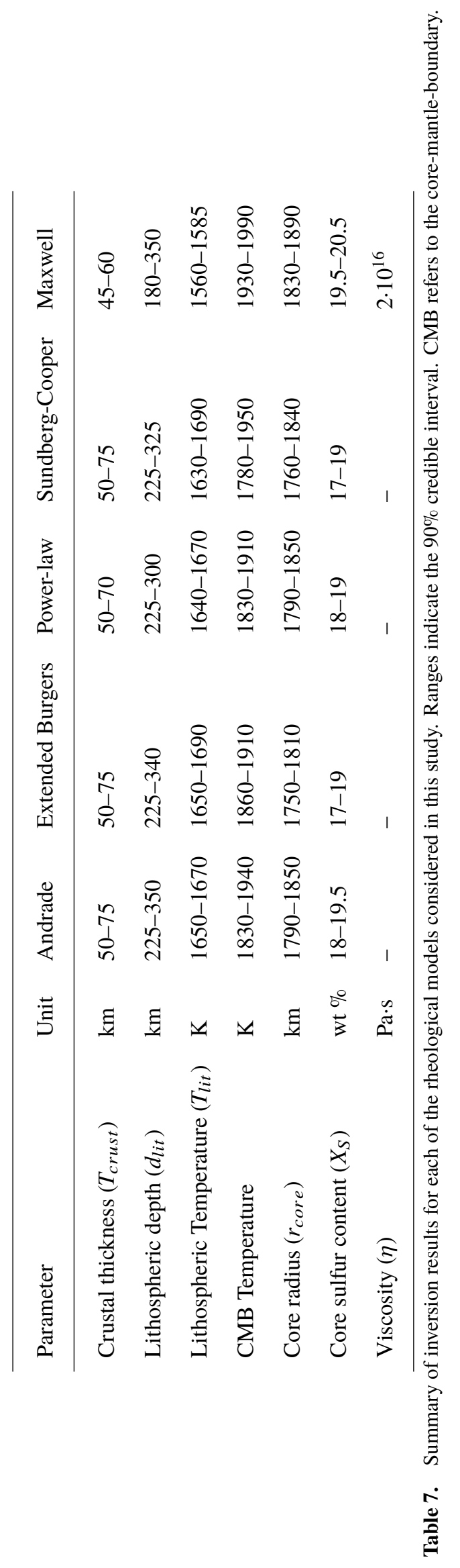




\begin{tabular}{|c|c|c|c|}
\hline Parameter & Value & Unit & Viscoelastic model \\
\hline$\beta$ & $3.2 \cdot 10^{-13}$ & $\mathrm{~Pa}^{-1} \mathrm{~s}^{-0.33}$ & $\mathrm{~A}$ \\
\hline$\beta$ & $0.5 \cdot 10^{-13}$ & $\mathrm{~Pa}^{-1} \mathrm{~s}^{-0.33}$ & $\mathrm{SC}$ \\
\hline$\Delta_{B}$ & 1.4 & - & ExtB, SC \\
\hline$\alpha$ & 0.33 & - & all \\
\hline$A$ & 0.002 & $\mathrm{~s}^{-0.33}$ & PL \\
\hline$d_{0}$ & 13.4 & $\mu \mathrm{m}$ & all \\
\hline$P_{0}$ & 0.2 & $\mathrm{GPa}$ & all \\
\hline$T_{0}$ & 1173 & $\mathrm{~K}$ & all \\
\hline$\tau_{L 0}$ & $10^{-3}$ & $\mathrm{~s}$ & ExtB, SC \\
\hline$\tau_{H 0}$ & $10^{7}$ & $\mathrm{~s}$ & ExtB, SC \\
\hline$\tau_{M 0}$ & $10^{7.48}$ & $\mathrm{~s}$ & all \\
\hline$\tau_{P 0}$ & $10^{-3.4}$ & $\mathrm{~s}$ & ExtB, SC \\
\hline$\Delta_{P}$ & 0.057 & - & ExtB, SC \\
\hline$m_{g a}$ & 1.3 & - & $\mathrm{A}, \mathrm{M}, \mathrm{ExtB}, \mathrm{SC}$ \\
\hline$m_{g v}$ & 3 & - & $\mathrm{A}, \mathrm{M}, \mathrm{ExtB}, \mathrm{SC}$ \\
\hline$V^{*}$ & $10^{-5}$ & $\mathrm{~m}^{3} / \mathrm{mol}$ & all \\
\hline$E^{*}$ & 360 & $\mathrm{~kJ} / \mathrm{mol}$ & all \\
\hline$\partial G / \partial P$ & 1.8 & - & all \\
\hline$\partial G / \partial T$ & -13.6 & $\mathrm{MPa} / \mathrm{K}$ & all \\
\hline$\sigma$ & 4 & - & ExtB, SC \\
\hline
\end{tabular}

Table A.1. Compilation of viscoelastic parameters used in this study. Abbreviations are: A-Andrade; ExtB-extended Burgers; M-Maxwell; PL-power-law; SC-Sundberg-Cooper. The values of $\partial G / \partial P$ and $\partial G / \partial T$ are only employed for creating the models discussed in section 2.3. All parameter values used are from Jackson and Faul [2010], except for $\beta$ and A (SC and PL), which are based on forward model runs such that the modeled $Q_{\mu}$ and $G_{R}$ (shown in Figure 3) among the various rheologies have comparable amplitudes. 


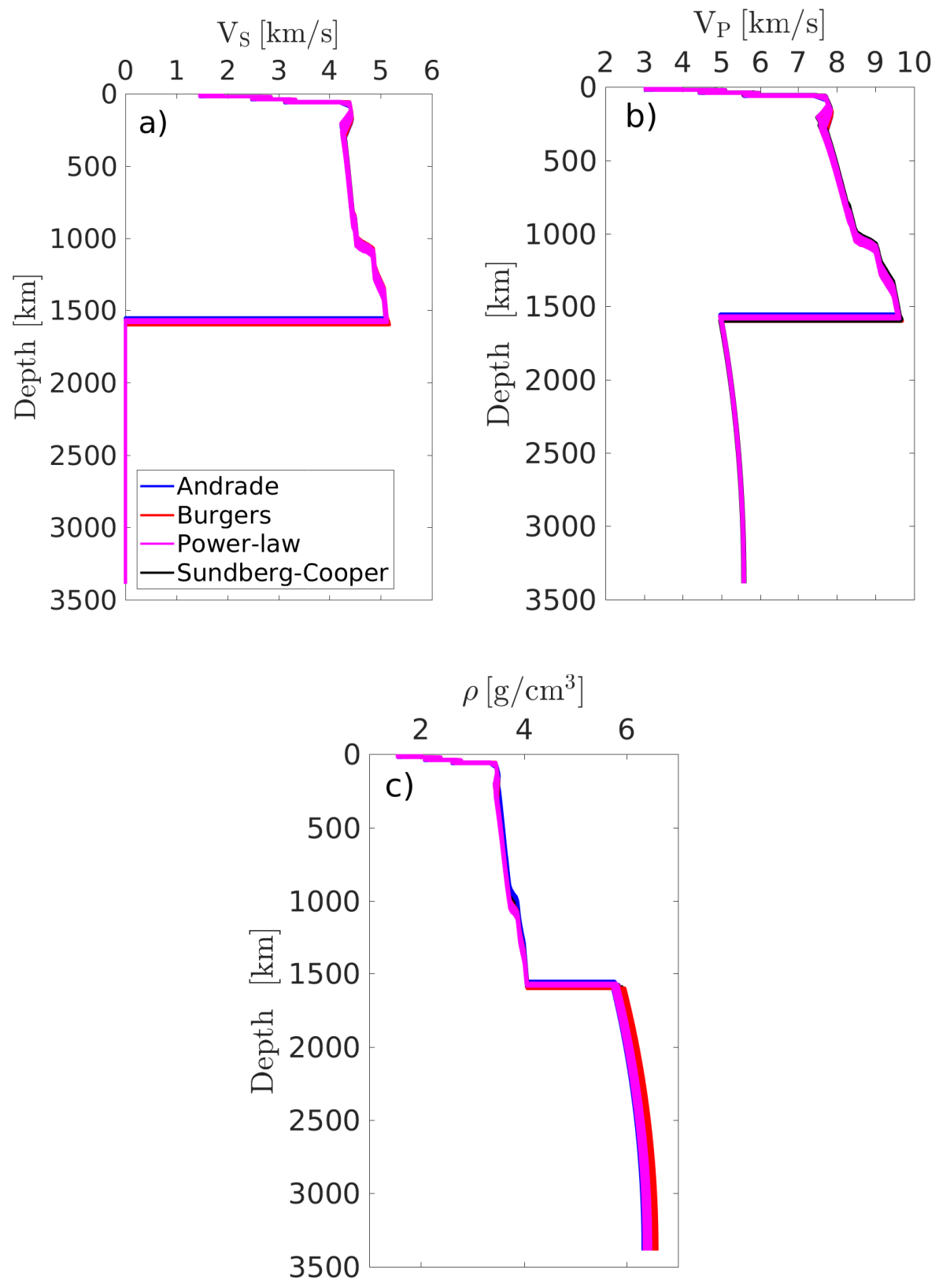

Figure C.1. Inverted seismic wave speed and density profiles obtained for each of the rheological models. a) P-wave speed $\left(V_{P}\right)$, b) S-wave speed $\left(V_{S}\right)$, and c) density $(\rho)$. 